\title{
THE LUMINOSITIES OF PROTOSTARS IN THE SPITZER c2d AND GOULD BELT LEGACY CLOUDS
}

\author{
Michael M. Dunham ${ }^{1}$, Héctor G. Arce ${ }^{1}$, Lori E. Allen ${ }^{2}$, Neal J. Evans II ${ }^{3}$, Hannah Broekhoven-Fiene ${ }^{4}$, \\ Nicholas L. Chapman ${ }^{5}$, Lucas A. Cieza ${ }^{6}$, Robert A. Gutermuth ${ }^{7}$, Paul M. Harvey ${ }^{3}$, Jennifer Hatchell $^{8}$, \\ Tracy L. Huard ${ }^{9}$, Jason M. Kirk ${ }^{10}$, Brenda C. Matthews ${ }^{4}$, Bruno Merín ${ }^{11}$, Jennifer F. Miller ${ }^{9,12}$, \\ Dawn E. Peterson ${ }^{13}$, AND LoRedana SPezzi ${ }^{14}$ \\ ${ }^{1}$ Department of Astronomy, Yale University, P.O. Box 208101, New Haven, CT 06520, USA; michael.dunham@yale.edu \\ ${ }^{2}$ National Optical Astronomy Observatories, Tucson, AZ, USA \\ ${ }^{3}$ Department of Astronomy, The University of Texas at Austin, 2515 Speedway, Stop C1400, Austin, TX 78712-1205, USA \\ ${ }^{4}$ Herzberg Institute, National Research Council of Canada, 5071 W. Saanich Road, Victoria, BC V9E 2E7, Canada \\ ${ }^{5}$ Center for Interdisciplinary Exploration and Research in Astrophysics (CIERA), Department of Physics \& Astronomy, \\ 2145 Sheridan Road, Evanston, IL 60208, USA \\ ${ }^{6}$ Institute for Astronomy, University of Hawaii at Manoa, Honolulu, HI 96822, USA \\ ${ }^{7}$ Department of Astronomy, University of Massachusetts, Amherst, MA, USA \\ ${ }^{8}$ Astrophysics Group, Physics, University of Exeter, Exeter EX4 4QL, UK \\ ${ }^{9}$ Department of Astronomy, University of Maryland, College Park, MD 20742, USA \\ ${ }^{10}$ School of Physics and Astronomy, Cardiff University, Queens Buildings, The Parade, Cardiff CF24 3AA, UK \\ ${ }^{11}$ Herschel Science Centre, ESAC-ESA, P.O. Box 78, E-28691 Villanueva de la Cañada, Madrid, Spain \\ ${ }^{12}$ Harvard-Smithsonian Center for Astrophysics, 60 Garden Street, Cambridge, MA 02138, USA \\ ${ }^{13}$ Space Science Institute, 4750 Walnut Street, Suite 205, Boulder, CO 80301, USA \\ ${ }^{14}$ European Southern Observatory (ESO), Karl-Schwarzschild-Strasse 2, D-85748 Garching bei München, Germany \\ Received 2012 October 4; accepted 2013 January 17; published 2013 February 28
}

\begin{abstract}
Motivated by the long-standing "luminosity problem" in low-mass star formation whereby protostars are underluminous compared to theoretical expectations, we identify 230 protostars in 18 molecular clouds observed by two Spitzer Space Telescope Legacy surveys of nearby star-forming regions. We compile complete spectral energy distributions, calculate $L_{\text {bol }}$ for each source, and study the protostellar luminosity distribution. This distribution extends over three orders of magnitude, from $0.01 L_{\odot}$ to $69 L_{\odot}$, and has a mean and median of $4.3 L_{\odot}$ and $1.3 L_{\odot}$, respectively. The distributions are very similar for Class 0 and Class I sources except for an excess of low luminosity $\left(L_{\mathrm{bol}} \lesssim 0.5 L_{\odot}\right)$ Class I sources compared to Class 0.100 out of the 230 protostars $(43 \%)$ lack any available data in the far-infrared and submillimeter $(70 \mu \mathrm{m}<\lambda<850 \mu \mathrm{m})$ and have $L_{\text {bol }}$ underestimated by factors of 2.5 on average, and up to factors of 8-10 in extreme cases. Correcting these underestimates for each source individually once additional data becomes available will likely increase both the mean and median of the sample by $35 \%-40 \%$. We discuss and compare our results to several recent theoretical studies of protostellar luminosities and show that our new results do not invalidate the conclusions of any of these studies. As these studies demonstrate that there is more than one plausible accretion scenario that can match observations, future attention is clearly needed. The better statistics provided by our increased data set should aid such future work.
\end{abstract}

Key words: stars: formation - stars: low-mass - stars: luminosity function, mass function - stars: protostars

\section{INTRODUCTION}

Low-mass stars form from the gravitational collapse of dense molecular cloud cores of gas and dust (e.g., Beichman et al. 1986; Di Francesco et al. 2007). During the collapse process material accretes from the core onto the protostar. In this paper the term protostar is used to refer to the hydrostatic object at the center of a collapsing core. More evolved young stellar objects (YSOs) no longer embedded within and forming from their natal dense cores are not considered protostars by this definition.

Despite several decades of progress, many details relating to the accretion of material from dense cores onto protostars remain poorly understood. As mass accretes onto protostars the gravitational energy is liberated and radiated away as accretion luminosity. This luminosity, which depends on the mass accretion rate, current protostellar mass, and current protostellar radius, can be used to study the mass accretion process and distinguish between different accretion models.

Observational studies of protostellar luminosities are hindered by the fact that protostars are deeply embedded in dense cores, with most of their emitted luminosities reprocessed to mid-infrared, far-infrared, and submillimeter wavelengths by the dust in the cores. The first significant study of the protostellar luminosity distribution was presented in a series of papers by Kenyon et al. $(1990,1994)$ and Kenyon \& Hartmann (1995). They identified 23 protostars in the Taurus-Auriga molecular cloud and calculated bolometric luminosities by integrating the observed spectral energy distributions (SEDs) using Infrared Astronomical Satellite (IRAS) $12-100 \mu \mathrm{m}$ photometry and longer-wavelength (sub)millimeter photometry from the ground, when available. They found that the protostellar luminosity distribution extended from 0.09 to $22 L_{\odot}$, with a mean and median of $2.3 L_{\odot}$ and $0.7 L_{\odot}$, respectively, and a strong peak around $0.3 L_{\odot}$.

As first noted by Kenyon et al. (1990), their observed protostellar luminosities are lower than expected from simple theoretical predictions. Their argument is as follows. First, they assumed that all observed luminosity is accretion luminosity,

$$
L_{\mathrm{acc}}=f_{\mathrm{acc}} \frac{G M \dot{M}_{\mathrm{acc}}}{R},
$$

where $f_{\text {acc }}$ is an efficiency factor taken to be $1, M$ and $R$ are the mass and radius of the protostar, and $\dot{M}_{\text {acc }}$ is the accretion 
rate onto the protostar. By further assuming that the peak of the luminosity distribution is produced by low-mass stars with $M=$ $0.1 M_{\odot}$ and $R=1 R_{\odot}$, they calculated an implied mass accretion rate of $\sim 10^{-7} M_{\odot} \mathrm{yr}^{-1}$. If some fraction of the observed luminosity arises from the protostar itself (contraction, deuterium burning, etc.), the implied mass accretion rate is even lower.

In the simplest model, the collapse of a singular isothermal sphere initially at rest as first considered by Shu (1977) and later extended by Terebey et al. (1984) to include rotation (often called the "standard model" of star formation), collapse proceeds in an "inside-out" fashion, beginning in the center of the core, moving outward at the sound speed, and giving rise to a constant mass accretion rate of $\dot{M}_{\text {acc }} \sim 2 \times 10^{-6} M_{\odot} \mathrm{yr}^{-1}$ for $10 \mathrm{~K}$ gas. This is over 10 times higher than inferred by Kenyon et al. (1990), and will only scale upward as $\dot{M}_{\text {acc }} \propto T^{3 / 2}$ for higher gas temperatures. Modifications to the standard model, including non-zero initial inward motions (Larson 1969; Penston 1969; Hunter 1977; Fatuzzo et al. 2004), magnetic fields (Galli \& Shu 1993a, 1993b; Li \& Shu 1997; Basu 1997), and isothermal spheres that are not singular but feature flattened density profiles at small radii (Foster \& Chevalier 1993; Henriksen et al. 1997) all tend to increase the accretion rate over that predicted by the standard model, making reconciliation between theory and the Kenyon et al. observations difficult. This has become known as the "luminosity problem."

Identification of protostars and determining their luminosities were both greatly improved by the launch of the Spitzer Space Telescope (Werner et al. 2004) in 2003. Many sites of star formation have been observed at wavelengths ranging from 3.6 to $160 \mu \mathrm{m}$ through various Spitzer surveys. One such survey was the Legacy survey "From Molecular Cores to Planet Forming Disks" (hereafter c2d; Evans et al. 2003), which observed 7 large, nearby molecular clouds and $\sim 100$ isolated dense cores and resulted in the discovery of very low luminosity objects (VeLLOs), protostars with internal luminosities $^{15} \leqslant 0.1 L_{\odot}$ embedded in dense cores (Young et al. 2004). Dunham et al. (2008) identified 15 VeLLOs in the c2d data set, and detailed studies of several have confirmed their very low luminosities and status as embedded protostars (Dunham et al. 2006, 2010a; Bourke et al. 2006; Lee et al. 2009; Kauffmann et al. 2011).

Both Enoch et al. (2009) and Evans et al. (2009) studied the c2d protostellar luminosity distribution by using the Spitzer data to identify protostars, compiling complete SEDs including far-infrared and (sub)millimeter photometry from the literature, and integrating these SEDs to determine $L_{\mathrm{bol}}$. They found a total of 112 protostars in the seven c2d clouds. Enoch et al. calculated mean and median values similar to those found by Kenyon \& Hartmann (1995), but with their improved sample statistics they noted the presence of a larger fraction of sources at low luminosities $\left(\lesssim 1.0 L_{\odot}\right)$. Evans et al. (2009) included a correction for foreground extinction and calculated revised mean and median values of $5.3 L_{\odot}$ and $1.5 L_{\odot}$. In a separate study, Kryukova et al. (2012) also derived the protostellar luminosity distribution for a number of star-forming clouds, including the c2d clouds. They found an even larger excess of low-luminosity protostars than found by Enoch et al. and Evans et al. Offner \& McKee (2011) argued that the higher observed luminosities found when extinction corrections are applied, combined with a more realistic value of the efficiency factor in Equation (1) of

\footnotetext{
15 The internal luminosity, $L_{\text {int }}$, is the luminosity of the central source and excludes luminosity arising from external heating.
}

$f_{\text {acc }} \sim 0.5$ to take into account both the powering of jets and winds and the effects of unseen, episodic accretion bursts, can essentially resolve the luminosity problem, although explaining the large fraction of sources at very low luminosities remains a challenge.

Several recent theoretical studies have explored possible resolutions to the luminosity problem, many of which were originally proposed by Kenyon et al. (1990). One possibility is that accretion is variable or episodic, with prolonged periods of low accretion punctuated by short bursts of rapid accretion. Numerous origins for such a process have been proposed, including gravitational instabilities in protostellar disks (e.g., Vorobyov \& Basu 2005, 2006, 2010; Machida et al. 2011; Cha \& Nayakshin 2011), a combination of gravitational and magneto-rotational instabilities in protostellar disks (e.g., Armitage et al. 2001; Zhu et al. 2009a, 2009b, 2010), quasi-periodic magnetically driven outflows in the envelope (Tassis \& Mouschovias 2005), decay and regrowth of magneto-rotational instability (MRI) turbulence (Simon et al. 2011), close interaction in binary systems or in dense stellar clusters (Bonnell \& Bastien 1992; Pfalzner et al. 2008), and disk-planet interactions (Lodato \& Clarke 2004; Nayakshin \& Lodato 2012). Indeed, Dunham \& Vorobyov (2012) showed that the $L_{\text {bol }}$ distribution predicted by the Vorobyov \& Basu (2005, 2006, 2010) simulations, which feature highly variable accretion with episodic bursts, provides a reasonable match to the $\mathrm{c} 2 \mathrm{~d}$ observations presented by Evans et al. (2009). Alternatively, Offner \& McKee (2011) presented analytic derivations of the protostellar luminosity function for several different accretion scenarios and showed that accretion models that tend toward a constant accretion time rather than a constant accretion rate provide a good match to the Evans et al. c2d observations. As a third alternative, Dalba \& Stahler (2012) recently argued that external accretion onto collapsing cores from the surrounding background cloud will reduce accretion rates and luminosities.

With 112 protostars spread over more than three orders of magnitude in $L_{\text {bol }}$, the c2d sample of protostellar luminosities is still somewhat limited by small number statistics. As a followup to c2d, the Spitzer Gould Belt Legacy Survey (hereafter GB; L. Allen et al., in preparation) observed most of the remaining clouds in the Gould Belt. In this paper we extend the identification of protostars and calculations of $L_{\mathrm{bol}}$ from Evans et al. (2009) to the combined c2d+GB data set. Our work is motivated by a desire for better underlying statistics in the observed protostellar luminosity distribution and improving the accuracy of the $L_{\mathrm{bol}}$ calculations by including additional data not yet available when the Evans et al. study was conducted. The organization of this paper is as follows. We describe our method in Section 2, including overviews of the c2d and GB surveys in Section 2.1, the identification of protostars in Section 2.2, the compilation of full source SEDs in Section 2.3, and the calculation of $L_{\mathrm{bol}}$ in Section 2.4. Section 3 summarizes our basic results. A discussion of these results is contained in Section 4. In particular, in Section 4.1 we compare our results to the existing c2d (Section 4.1.1) and Kryukova et al. (2012) (Section 4.1.2) results, in Section 4.2 we discuss several recent theoretical investigations of protostellar luminosities, and in Section 4.3 we evaluate the accuracy of our $L_{\text {bol }}$ measurements for sources with observed SEDs that are not well sampled in the far-infrared and submillimeter, and the effects of this incomplete sampling on our overall results. Finally, we outline important future work needed to further advance this topic in Section 5, and summarize our findings in Section 6. 
Table 1

Molecular Clouds Surveyed by the c2d and GB Surveys

\begin{tabular}{|c|c|c|c|c|}
\hline Cloud & Survey & $\begin{array}{c}\text { Distance } \\
\text { (pc) }\end{array}$ & $\begin{array}{c}\text { Distance } \\
\text { Reference }^{\mathrm{a}}\end{array}$ & Data Reference $(\mathrm{s})^{\mathrm{b}}$ \\
\hline Aquila & GB & 260 & Maury et al. (2011) & Gutermuth et al. (2008); Maury et al. (2011) \\
\hline Auriga/California & GB & 450 & Lada et al. (2009) & H. Broekhoven-Fiene et al. (in preparation) \\
\hline Cepheus & GB & $200-325^{\mathrm{c}}$ & Kirk et al. (2009) & Kirk et al. (2009) \\
\hline Chamaeleon I & GB & 150 & Belloche et al. (2011b) & $\ldots$ \\
\hline Chamaeleon II & $\mathrm{c} 2 \mathrm{~d}$ & 178 & Whittet et al. (1997) & Young et al. (2005); Porras et al. (2007); Alcalá et al. (2008) \\
\hline Chamaeleon III & GB & 150 & Belloche et al. (2011b) & $\ldots$ \\
\hline Corona Australis & GB & 130 & Neuhäuser \& Forbrich (2008) & Peterson et al. (2011) \\
\hline IC5146 & GB & 950 & Harvey et al. (2008) & Harvey et al. (2008) \\
\hline Lupus I & $\mathrm{c} 2 \mathrm{~d}$ & 150 & Comerón (2008) & Chapman et al. (2007); Merín et al. (2008) \\
\hline Lupus III & $\mathrm{c} 2 \mathrm{~d}$ & 200 & Comerón (2008) & Chapman et al. (2007); Merín et al. (2008) \\
\hline Lupus IV & $\mathrm{c} 2 \mathrm{~d}$ & 150 & Comerón (2008) & Chapman et al. (2007); Merín et al. (2008) \\
\hline Lupus V & GB & 150 & Comerón (2008) & Spezzi et al. (2011) \\
\hline Lupus VI & GB & 150 & Comerón (2008) & Spezzi et al. (2011) \\
\hline Musca & GB & 160 & Knude \& Hog (1998) & T. Huard et al. (in preparation) \\
\hline Ophiuchus & $\mathrm{c} 2 \mathrm{~d}$ & 125 & de Geus et al. (1989) & Padgett et al. (2008) \\
\hline Ophiuchus North & GB & 130 & Wilking et al. (2008) & Hatchell et al. (2012) \\
\hline Perseus & $\mathrm{c} 2 \mathrm{~d}$ & 250 & Enoch et al. (2006) & Jørgensen et al. (2006); Rebull et al. (2007) \\
\hline Serpens & $\mathrm{c} 2 \mathrm{~d}$ & 429 & Dzib et al. $(2010,2011)$ & Harvey et al. (2006, 2007a, 2007b) \\
\hline
\end{tabular}

Notes.

${ }^{a}$ Reference for the distance quoted in this work.

b References presenting the Spitzer/IRAC and MIPS observations.

${ }^{c}$ Different regions within Cepheus are located at different distances; see Kirk et al. (2009) for details.

\section{METHOD}

\subsection{Overview of the Surveys}

The Spitzer c2d survey (PI: N. J. Evans) conducted an imaging survey of seven large, nearby molecular clouds and about 100 isolated molecular cloud cores, and a spectroscopic survey of selected targets. The science questions motivating this survey and a summary of the observation strategy are given by Evans et al. (2003). A summary of the results from the survey of the large molecular clouds is given by Evans et al. (2009). The Spitzer GB survey (PI: L. E. Allen) was designed as a follow-up to the clouds portion of $\mathrm{c} 2 \mathrm{~d}$ and conducted an imaging survey of 11 nearby molecular clouds, completing most of the remaining clouds in the Gould Belt (L. Allen et al., in preparation; see also Gutermuth et al. 2008; Harvey et al. 2008; Kirk et al. 2009; Peterson et al. 2011; Spezzi et al. 2011; Hatchell et al. 2012). The two surveys obtained 3.6-8.0 $\mu \mathrm{m}$ images with the Spitzer Infrared Array Camera (IRAC; Fazio et al. 2004) and 24-160 $\mu \mathrm{m}$ images with the Multiband Imaging Photometer (MIPS; Rieke et al. 2004) of all 18 clouds. A standard pipeline developed by $\mathrm{c} 2 \mathrm{~d}$ was used for data reduction, source extraction, and band-merging to produce final source catalogs for both surveys and has been described in detail elsewhere (Harvey et al. 2006; Evans et al. 2007).

Table 1 lists each cloud, the survey in which it was imaged (c2d or GB), the assumed distance to the cloud, the reference for the distance, and references of individual studies of each cloud where the observation strategy and basic results are presented. These clouds were chosen to represent nearly all of the significant sites of star formation within the Gould Belt, with two major exceptions: the Taurus and Orion molecular clouds. These two clouds were each the focus of separate, dedicated Spitzer Legacy surveys led by other groups, and folding in their results with the $\mathrm{c} 2 \mathrm{~d}+\mathrm{GB}$ clouds will be the focus of a future paper. The clouds listed in Table 1 span very large ranges of properties. For example, the total cloud masses range from a few hundred $M_{\odot}$ (e.g., Chamaeleon II; Evans et al. 2009) to $\sim 10^{5} M_{\odot}$ (Auriga/California Molecular Cloud; Lada et al. 2009), the star formation rates and star formation rate surface densities both span approximately two orders of magnitude (Evans et al. 2009; Heiderman et al. 2010), and the ratio of protostars to pre-main sequence stars (indicative of the amount of current star formation still on-going in the cloud) range from none (e.g., Lupus V and VI; Spezzi et al. 2011) to values in excess of $30 \%$ (e.g., Auriga/California Molecular Cloud, Cepheus, IC5146, and Perseus; H. Broekhoven-Fiene et al., in preparation; Kirk et al. 2009; Harvey et al. 2008; Evans et al. 2009). We refer the reader to the individual cloud studies listed in Table 1 for more details and additional references.

We caution that the distances to the 18 clouds surveyed are not all well-known, and some cloud distances are still under significant debate. One such example is the debate over the distance(s) to the Serpens and Aquila regions. Recent Very Long Baseline Array parallax measurements led to a $65 \%$ increase in the distance to Serpens compared to that assumed by the c2d team (429 versus 260 pc; Straižys et al. 1996; Harvey et al. 2006; Dzib et al. 2010, 2011), and there remains debate whether or not Aquila is also located at this new, farther distance or even if all of Aquila is itself located at the same distance (e.g., Gutermuth et al. 2008; Maury et al. 2011). We do not list formal distance uncertainties in Table 1 as such uncertainties are very poorly characterized in at least some clouds. Instead we refer to the references listed in Table 1 for detailed discussions on the various methods used to derive distances and the uncertainties in these methods. Future distance revisions will require future revisions to the results presented in this study.

\subsection{Sample Selection}

Our method for selecting protostars from the c2d and GB observations closely follows the selection method used by Evans et al. (2009) for the c2d clouds. We summarize the main points here and refer to Evans et al. for more details. 
The data reduction pipeline creates band-merged source catalogs incorporating Two Micron All Sky Survey (2MASS) and Spitzer $1.25-70 \mu \mathrm{m}$ photometry for each cloud. Candidate YSOs are identified using a standard classification method developed for the Spitzer c2d and GB projects. This method is described in detail in Harvey et al. (2007a) and Evans et al. (2007) and summarized in all of the publications presenting individual cloud studies listed in Table 1. Briefly, this method uses the Spitzer SWIRE Legacy survey of the ELAIS N1 extragalactic field (Lonsdale et al. 2003), processed to simulate the sensitivity and extinction distribution of the clouds in the $\mathrm{c} 2 \mathrm{~d}$ and GB surveys, to determine the positions of galaxies in three different Spitzer color-magnitude diagrams. Each source extracted in the $\mathrm{c} 2 \mathrm{~d}$ and $\mathrm{GB}$ cloud catalogs with infrared colors indicative of the presence of dust (sources with colors that can not be explained by extincted background stars) is then assigned an unnormalized "probability" of being a galaxy or YSO based on its position in each color-magnitude diagram, its $K-$ [4.5] color, whether it was found to be extended in either of the two shortest Spitzer/IRAC bands (3.6 and $4.5 \mu \mathrm{m})$, and its flux density at 24 and $70 \mu \mathrm{m}$. The color and magnitude boundaries, along with the final boundary between candidate YSO and candidate galaxy in unnormalized "probability," are set to provide a nearly complete elimination of SWIRE sources. We refer the reader to Harvey et al. (2007a) for further details on this classification method. Similar classification methods have been presented by other Spitzer studies of galactic starforming regions (e.g., Gutermuth et al. 2009; Rebull et al. 2010; Kryukova et al. 2012).

In total, we identified 3239 candidate YSOs in the $18 \mathrm{c} 2 \mathrm{~d}$ and GB catalogs. All sources were visually inspected to remove residual contaminants, including resolved galaxies misclassified as candidate YSOs and image artifacts identified as pointsources by the automated pipeline but lacking true point-source detections in one or more bands (see Evans et al. 2009 for details). Follow-up optical spectroscopy of targets in Serpens presented by Oliveira et al. (2009) led to the identification and removal of 11 background giants with infrared excesses. We lack the data required to identify and remove such objects in the other clouds. Oliveira et al. (2009) found a contamination rate of $25 \%$ in their Serpens study. Serpens (and Aquila) are likely the worst cases due to their close proximity to the Galactic plane (spanning Galactic latitudes ranging from $2^{\circ}$ to $10^{\circ}$ ), although Romero et al. (2012) recently suggested the contamination rate is at least as high in other clouds as well, and Hatchell et al. (2012) found that $27 \%$ of their sample of candidate YSOs in Ophiuchus North selected via the c2d criteria were likely to be background giants based on proper motion arguments. However, $80 \%$ of the contaminants identified by Oliveira et al. and $75 \%$ of the contaminants identified by Hatchell et al. are classified as Class III YSOs, thus even if the overall contamination rate is as high as $25 \%-30 \%$, our inability to remove these contaminants will not significantly affect this study since it is only focused on the subset of YSOs that are considered to be protostars. Finally, a few known YSOs missing from the list of candidate YSOs due to missing photometry at one or more Spitzer wavelengths caused by saturation or nondetections from being too deeply embedded were added by hand.

The above process resulted in a final list of 2966 YSOs (since all 2966 sources passed visual inspection, we have followed the terminology used by Evans et al. (2009) and dropped the word "candidate" at this point). This is nearly a factor of three increase over the 1024 YSOs identified in the $\mathrm{c} 2 \mathrm{~d}$ clouds alone by Evans et al. (2009). Many of these YSO populations have already been presented and discussed in detailed studies of individual clouds (see Table 1 for references) and in an analysis of the star formation rates and efficiencies of the c2d and GB clouds based on a preliminary version of the final YSO catalog (Heiderman et al. 2010). A complete analysis of the full YSO population, implications for star formation rates and efficiencies in the Gould Belt, and the evolution and lifetimes of YSOs will be presented in a forthcoming paper (L. Allen et al., in preparation). Here we focus only on the observed luminosities of protostars.

The final sample of protostars is identified from the list of 2966 YSOs by examining the full SEDs compiled for each source (see below) and selecting only those sources associated with at least one (sub)millimeter detection at $\lambda \geqslant 350 \mu \mathrm{m}$, resulting in a final sample of 230 protostars. This is identical to the procedure followed by Evans et al. (2009) except they used a cutoff wavelength of $850 \mu \mathrm{m}$; we modified this to $350 \mu \mathrm{m}$ because of the large increase in available data at this wavelength. No intrinsic protostellar colors were assumed and no additional color criteria were imposed. This decision is motivated by numerous recent studies that have used dust radiative transfer models to show that protostars observed through outflow cavities can resemble more evolved Class II or Class III sources in the infrared (e.g., Whitney et al. 2003; Robitaille et al. 2006; Crapsi et al. 2008; Dunham et al. 2010b). By selecting all sources associated with (sub)millimeter detections we recover such sources and identify all YSOs that are associated with dense cores, although future follow-up observations are required to remove true Class II or III sources simply seen in projection against a dense core.

By requiring a (sub)millimeter detection, our method requires the availability of (sub)millimeter surveys covering the full extents of the clouds surveyed by $\mathrm{c} 2 \mathrm{~d}$ and GB. This is not always the case, as described in more detail in the next section below. The effects of this limitation will be discussed in detail in Section 4.1.2, where we compare to a recent study that used very different methods for selecting protostars and did not require (sub)millimeter detections.

\subsection{Constructing Full SEDs and Correcting for Extinction}

Similar to Evans et al. (2009), we compiled as complete SEDs as possible for each of the 2966 YSOs. In addition to the 2MASS and Spitzer $1.25-70 \mu \mathrm{m}$ photometry provided by the source catalogs, we included the following: (1) optical photometry, where available from the literature, (2) Wide-field Infrared Survey Explorer (WISE) 12 and $22 \mu \mathrm{m}$ photometry from the all-sky catalog, ${ }^{16}$ (3) selected other ground-based optical and infrared data as compiled by the authors of the detailed studies of individual clouds (see references in Table 1), (4) Spitzer $160 \mu \mathrm{m}$ photometry for sources detected and not located in saturated or confused regions, calculated using aperture photometry and aperture corrections as given by the MIPS Instrument Handbook, ${ }^{17}$ (5) SHARC-II ${ }^{18} 350 \mu \mathrm{m}$ photometry, when available, from a targeted survey of protostellar sources (Wu et al. 2007;

\footnotetext{
16 Available at http://irsa.ipac.caltech.edu/cgi-bin/Gator/nphscan?mission $=$ irsa\&submit $=$ Select $\&$ projshort $=$ WISE .

17 Available at http://irsa.ipac.caltech.edu/data/SPITZER/docs/mips/ mipsinstrumenthandbook/.

18 The Submillimeter High Angular Resolution Camera II (SHARC-II) is a $350 \mu \mathrm{m}$ bolometer array operated at the Caltech Submillimeter Observatory (Dowell et al. 2003).
} 
M. M. Dunham et al., in preparation), (6) SCUBA ${ }^{19} 450$ and $850 \mu \mathrm{m}$ photometry, when available, from the SCUBA Legacy Catalog (Di Francesco et al. 2008), and (7) other (sub)millimeter photometry from unbiased surveys of molecular clouds, where available.

For the last item above, other (sub)millimeter photometry from unbiased surveys of molecular clouds, we used photometry from the following surveys: (1) A MAMBO2 ${ }^{20} 1.2 \mathrm{~mm}$ survey of part of Aquila (Maury et al. 2011); (2) A LABOCA ${ }^{21} 870 \mu \mathrm{m}$ survey of Chamaeleon I (Belloche et al. 2011b); (3) A SIMBA 22 $1.2 \mathrm{~mm}$ survey of Chamaeleon II (Young et al. 2005); (4) A LABOCA $870 \mu \mathrm{m}$ survey of Chamaeleon III (Belloche et al. 2011a); (5) A Bolocam ${ }^{23} 1.1 \mathrm{~mm}$ survey of Ophiuchus (Young et al. 2006); (6) A Bolocam $1.1 \mathrm{~mm}$ survey of Perseus (Enoch et al. 2006); and (7) A Bolocam $1.1 \mathrm{~mm}$ survey of Serpens (Enoch et al. 2007).

Summarizing the above information, we have access to complete (sub)millimeter surveys for only 6 out of the 18 clouds (Chamaeleon I, Chamaeleon II, Chamaeleon III, Ophiuchus, Perseus, and Serpens), plus a partial survey of Aquila and piecemeal coverage of other clouds from the SCUBA Legacy Catalog (Di Francesco et al. 2008). This incomplete (sub)millimeter coverage will affect both our luminosity calculations and ability to identify protostars, and these effects are discussed in detail in Sections 4.3 and 5.1.

Finally, before using the SEDs to calculate bolometric luminosities, we correct the photometry for foreground extinction. We wish to only correct for the foreground cloud extinction and not the local extinction from the dense core itself, as in the latter case the extincted emission is reprocessed to longer wavelengths and included in our observed SEDs. Determining the true lineof-sight extinction to a protostar from the foreground cloud is not a trivial task. Following Evans et al. (2009), we assign extinction values to all 2966 YSOs (a sample which includes the 230 protostars identified in this work) as follows.

1. We adopt extinction values from the literature for Class II and III YSOs (classified via infrared spectral index; see Evans et al. 2009 for details) included in published optical studies.

2. We de-redden the remaining Class II and III YSOs to the intrinsic near-infrared colors of an assumed spectral type of $\mathrm{K} 7$, found to be fairly representative of the majority of Class II and III YSOs in the c2d clouds (Oliveira et al. 2009, 2010; see also Evans et al. 2009 for details).

3. We de-redden all of the Class I and Flat spectrum YSOs (again classified via infrared spectral index) in each cloud using the mean extinction toward all Class II YSOs in that cloud.

The extinction values adopted for each of our 230 protostars following this procedure are listed in Table 2. Most of the protostars in a given cloud have the same adopted extinction value since most protostars are classified as Class I or flat

\footnotetext{
19 The Submillimeter Common-User Bolometer Array (SCUBA) was a 450 and $850 \mu \mathrm{m}$ bolometer array operated at the James Clerk Maxwell Telescope.

20 The Max-Planck Millimeter Bolometer 2 (MAMBO2) was a $1.2 \mathrm{~mm}$ bolometer array operated at the IRAM $30 \mathrm{~m}$ telescope.

21 The Large Apex Bolometer Camera (LABOCA) is an $870 \mu \mathrm{m}$ bolometer array in operation at the Atacama Pathfinder Experiment telescope (Siringo et al. 2009).

22 The SEST Imaging Bolometer Array was a $1.2 \mathrm{~mm}$ bolometer array in operation at the Swedish-ESO Submillimeter Telescope.

23 Bolocam is a 1.1 and $2.1 \mathrm{~mm}$ bolometer array operated at the Caltech Submillimeter Observatory (Glenn et al. 1998).
}

spectrum via their infrared spectral index, although some have different values since no intrinsic protostellar colors were assumed by our selection criteria and thus some Class II YSOs are classified as protostars (see Section 2.2 above).

Once the extinction values are assigned, we use these values combined with the Weingartner \& Draine (2001) extinction law for $R_{V}=5.5$ to correct the photometry for extinction. The choice of the $R_{V}=5.5$ law rather than the $R_{V}=3.1$ law is motivated by several studies showing that the former is more appropriate for the dense regions in which stars form (e.g., Chapman et al. 2009). While we do caution that our approach is somewhat crude, it is the best that can currently be done and is significantly more reliable than ignoring the effects of extinction altogether.

\subsection{Calculation of Evolutionary Indicators}

Once we have constructed full SEDs as described above in Section 2.3, we use these SEDs to calculate the bolometric luminosities $\left(L_{\mathrm{bol}}\right)$ and bolometric temperatures $\left(T_{\mathrm{bol}}\right) . L_{\mathrm{bol}}$ is calculated by integrating over all detections,

$$
L_{\mathrm{bol}}=4 \pi d^{2} \int_{0}^{\infty} S_{\nu} d \nu .
$$

The bolometric temperature is defined to be the temperature of a blackbody with the same flux-weighted mean frequency as the source (Myers \& Ladd 1993). Following Myers \& Ladd, $T_{\text {bol }}$ is calculated as

$$
T_{\text {bol }}=1.25 \times 10^{-11} \frac{\int_{0}^{\infty} v S_{\nu} d v}{\int_{0}^{\infty} S_{\nu} d v} \mathrm{~K} .
$$

$T_{\text {bol }}$ can be thought of as a protostellar equivalent of $T_{\text {eff }}$ for stars; $T_{\text {bol }}$ starts at very low values $(\sim 10 \mathrm{~K})$ for cold, starless cores and eventually increases to $T_{\text {eff }}$ once the core and disk have fully dissipated. The integrals defined in Equations (2) and (3) are calculated using the trapezoid rule to integrate over the finitely sampled SEDs. To avoid model or fitting uncertainties and focus only on the observations themselves, we do not extrapolate beyond the shortest and longest frequencies at which data are available and we do not interpolate over missing data. Instead, we explore the effects of missing data on our $L_{\text {bol }}$ calculations in Section 4.3. We calculate $L_{\text {bol }}$ and $T_{\text {bol }}$ twice, once with the original, observed photometry and once with the extinctioncorrected photometry.

\section{RESULTS}

For each of the 230 protostars identified following the selection method described above, Table 2 lists a running index, the cloud in which the protostar is located, the Spitzer source name (which also gives the coordinates), the assumed $A_{V}$ for extinction corrections, the infrared spectral index ${ }^{24}(\alpha), T_{\text {bol }}$, and $L_{\text {bol }}$ calculated from both the observed and extinction corrected photometry, and a flag indicating whether or not each protostar has any available data at $70 \mu \mathrm{m}<\lambda<850 \mu \mathrm{m}$ (see Section 4.3). In Table 2 the extinction corrected values are denoted as $\alpha^{\prime}$, $T_{\text {bol }}^{\prime}$, and $L_{\text {bol }}^{\prime}$ to differentiate them from the observed values. Throughout the remainder of this paper we consider only the extinction corrected values and drop the primes for simplicity. We do not give uncertainties for the $L_{\text {bol }}$ derived in this work.

\footnotetext{
24 The infrared spectral index, $\alpha$, is calculated over all 2MASS and Spitzer
} detections from 2-24 $\mu \mathrm{m}$ (Evans et al. 2007). 
Table 2

List of Protostars and Basic Properties

\begin{tabular}{|c|c|c|c|c|c|c|c|c|c|c|}
\hline \multirow[t]{2}{*}{ Index } & \multirow[t]{2}{*}{ Cloud } & \multirow{2}{*}{$\begin{array}{c}\text { Spitzer } \\
\text { Source Name } \\
(\mathrm{SSTc} 2 \mathrm{~d} \text { or SSTgb }+)\end{array}$} & \multirow[t]{2}{*}{$A_{V}{ }^{\mathrm{a}}$} & \multicolumn{3}{|c|}{ Observed } & \multicolumn{3}{|c|}{ Extinction Corrected } & \multirow[t]{2}{*}{ FIR/SMM ${ }^{b}$} \\
\hline & & & & $\alpha$ & $\begin{array}{l}T_{\text {bol }} \\
(\mathrm{K})\end{array}$ & $\begin{array}{c}L_{\mathrm{bol}} \\
\left(L_{\odot}\right)\end{array}$ & $\alpha^{\prime}$ & $\begin{array}{c}T_{\text {bol }}{ }^{\prime} \\
(\mathrm{K})\end{array}$ & $\begin{array}{l}L_{\mathrm{bol}}{ }^{\prime} \\
\left(L_{\odot}\right)\end{array}$ & \\
\hline 1 & Aquila & J1829053-014157 & 12.4 & 1.14 & 80 & 2.7 & 0.96 & 96 & 2.9 & $\mathrm{~N}$ \\
\hline 2 & Aquila & $\mathrm{J} 1829234-013856$ & 12.4 & 1.39 & 250 & 0.84 & 1.08 & 320 & 1.2 & $\mathrm{~N}$ \\
\hline 3 & Aquila & $\mathrm{J} 1829381-015101$ & 12.4 & 1.30 & 240 & 0.33 & 1.09 & 260 & 0.46 & $\mathrm{~N}$ \\
\hline 4 & Aquila & $\mathrm{J} 1829387-015100$ & 12.4 & 0.82 & 84 & 3.0 & 0.65 & 110 & 3.3 & $\mathrm{~N}$ \\
\hline 5 & Aquila & $\mathrm{J} 1829419-015012$ & 12.4 & 0.65 & 110 & 0.067 & 0.46 & 150 & 0.078 & $\mathrm{~N}$ \\
\hline 6 & Aquila & $\mathrm{J} 1829433-015652$ & 12.4 & 0.81 & 310 & 0.67 & 0.94 & 380 & 0.99 & $\mathrm{~N}$ \\
\hline 7 & Aquila & $\mathrm{J} 1829470-015548$ & 12.4 & 0.60 & 220 & 0.85 & 0.29 & 330 & 1.1 & $\mathrm{~N}$ \\
\hline 8 & Aquila & J1829595-020106 & 12.4 & 0.74 & 180 & 0.037 & 0.53 & 230 & 0.049 & $\mathrm{~N}$ \\
\hline 9 & Aquila & $\mathrm{J} 1830011-020609$ & 12.4 & 0.76 & 110 & 0.85 & 0.34 & 160 & 0.98 & $\mathrm{~N}$ \\
\hline 10 & Aquila & $\mathrm{J} 1830025-020258$ & 12.4 & 0.48 & 390 & 1.2 & 0.36 & 470 & 1.9 & $\mathrm{~N}$ \\
\hline 11 & Aquila & $\mathrm{J} 1830175-020958$ & 12.4 & 1.32 & 130 & 0.24 & 1.11 & 150 & 0.30 & $\mathrm{~N}$ \\
\hline 12 & Aquila & $\mathrm{J} 1830246-015411$ & 12.4 & 0.21 & 400 & 0.73 & 0.12 & 590 & 1.1 & $\mathrm{~N}$ \\
\hline 13 & Aquila & $\mathrm{J} 1830259-021043$ & 12.4 & 1.38 & 95 & 3.5 & 1.20 & 110 & 4.0 & $\mathrm{~N}$ \\
\hline 14 & Aquila & $\mathrm{J} 1830293-015643$ & 12.4 & 0.21 & 480 & 1.5 & -0.06 & 710 & 2.6 & $\mathrm{~N}$ \\
\hline 15 & Aquila & $\mathrm{J} 1830469-015646$ & 12.4 & 1.18 & 120 & 0.16 & 0.87 & 140 & 0.20 & $\mathrm{~N}$ \\
\hline 16 & Aquila & $\mathrm{J} 1830487-015602$ & 12.4 & 0.98 & 190 & 0.28 & 0.80 & 250 & 0.36 & $\mathrm{~N}$ \\
\hline 17 & Aquila & $\mathrm{J} 1831522-020126$ & 12.4 & 1.56 & 160 & 0.12 & 1.35 & 170 & 0.16 & $\mathrm{~N}$ \\
\hline 18 & Aquila & $\mathrm{J} 1832132-015730$ & 12.4 & 0.93 & 57 & 0.28 & 0.73 & 59 & 0.29 & $\mathrm{~N}$ \\
\hline 19 & Auriga/California & J0410416+380805 & 10.0 & -0.32 & 50 & 7.0 & -0.88 & 98 & 7.3 & $\mathrm{Y}$ \\
\hline 20 & Auriga/California & $\mathrm{J} 0430036+351420$ & 7.5 & 0.75 & 98 & 0.75 & 0.80 & 140 & 0.80 & $\mathrm{~N}$ \\
\hline 21 & Auriga/California & $\mathrm{J} 0430082+351410$ & 7.5 & 0.46 & 130 & 0.81 & 0.44 & 180 & 0.90 & $\mathrm{~N}$ \\
\hline 22 & Auriga/California & J0430145+351332 & 23.4 & -0.39 & 720 & 0.94 & -1.26 & 1500 & 4.7 & $\mathrm{~N}$ \\
\hline 23 & Cepheus & $\mathrm{J} 2035463+675302$ & 5.4 & 1.00 & 50 & 1.4 & 0.56 & 53 & 1.4 & $\mathrm{Y}$ \\
\hline 24 & Cepheus & J2036198+675631 & 6.5 & -0.50 & 760 & 2.2 & -0.64 & 2500 & 4.9 & $\mathrm{Y}$ \\
\hline 25 & Cepheus & $\mathrm{J} 2040567+672305$ & 5.4 & 0.81 & 150 & 0.11 & 0.30 & 200 & 0.12 & $\mathrm{~N}$ \\
\hline 26 & Cepheus & $\mathrm{J} 2057130+773543$ & 5.4 & 0.10 & 360 & 2.0 & -0.04 & 530 & 2.4 & $\mathrm{Y}$ \\
\hline 27 & Cepheus & $\mathrm{J} 2100207+681316$ & 5.4 & 1.60 & 89 & 0.90 & 1.36 & 100 & 0.95 & $\mathrm{Y}$ \\
\hline 28 & Cepheus & $\mathrm{J} 2100221+681258$ & 5.4 & 0.95 & 87 & 0.83 & 0.52 & 100 & 0.86 & $\mathrm{Y}$ \\
\hline 29 & Cepheus & $\mathrm{J} 2101328+681120$ & 5.4 & 1.00 & 21 & 3.1 & 0.94 & 26 & 3.1 & $\mathrm{Y}$ \\
\hline 30 & Cepheus & $\mathrm{J} 2102212+675420$ & 5.4 & 1.10 & 180 & 0.41 & 0.64 & 210 & 0.44 & $\mathrm{Y}$ \\
\hline 31 & Cepheus & $\mathrm{J} 2102273+675418$ & 5.4 & -0.29 & 36 & 0.32 & -0.64 & 47 & 0.33 & $\mathrm{Y}$ \\
\hline 32 & Cepheus & $\mathrm{J} 2228030+690116$ & 5.4 & 0.84 & 160 & 2.1 & 0.40 & 180 & 2.3 & $\mathrm{Y}$ \\
\hline 33 & Cepheus & $\mathrm{J} 2228074+690038$ & 5.4 & 1.00 & 42 & 0.94 & 0.90 & 43 & 0.95 & $\mathrm{Y}$ \\
\hline 34 & Cepheus & $\mathrm{J} 2229333+751316$ & 5.4 & 0.12 & 210 & 0.086 & 0.10 & 240 & 0.096 & $\mathrm{~N}$ \\
\hline 35 & Cepheus & $\mathrm{J} 2229594+751403$ & 5.4 & 0.49 & 270 & 0.32 & 0.16 & 330 & 0.36 & $\mathrm{~N}$ \\
\hline 36 & Cepheus & $\mathrm{J} 2230318+751409$ & 5.4 & 0.71 & 35 & 0.43 & 0.64 & 36 & 0.43 & $\mathrm{Y}$ \\
\hline 37 & Cepheus & $\mathrm{J} 2231056+751337$ & 5.1 & -0.51 & 30 & 0.30 & -0.77 & 31 & 0.30 & $\mathrm{Y}$ \\
\hline 38 & Cepheus & $\mathrm{J} 2238428+751136$ & 5.4 & 1.20 & 72 & 1.8 & 0.89 & 78 & 1.8 & $\mathrm{Y}$ \\
\hline 39 & Cepheus & $\mathrm{J} 2238469+751133$ & 5.4 & 1.60 & 110 & 6.2 & 1.94 & 120 & 6.6 & $\mathrm{Y}$ \\
\hline 40 & Cepheus & $\mathrm{J} 2238530+751123$ & 5.4 & 0.97 & 90 & 1.4 & 0.44 & 150 & 1.5 & $\mathrm{Y}$ \\
\hline 41 & Cepheus & $\mathrm{J} 2039062+680215$ & 5.4 & 0.94 & 35 & 4.0 & 0.79 & 35 & 4.1 & $\mathrm{Y}$ \\
\hline 42 & Cepheus & $\mathrm{J} 2045539+675738$ & 5.4 & $\ldots$ & 340 & 48 & $\ldots$ & 450 & 62 & $\mathrm{Y}$ \\
\hline 43 & Cepheus & $\mathrm{J} 2235234+751707$ & 5.4 & 2.40 & 230 & 8.7 & 3.69 & 280 & 9.5 & $\mathrm{Y}$ \\
\hline 44 & Chamaeleon I & J1104227-771808 & 6.6 & 0.61 & 350 & 0.086 & 0.27 & 510 & 0.11 & $\mathrm{~N}$ \\
\hline 45 & Chamaeleon I & J1106464-772232 & 6.6 & 1.23 & 66 & 0.69 & 1.11 & 74 & 0.72 & $\mathrm{Y}$ \\
\hline 46 & Chamaeleon I & $\mathrm{J} 1106580-772248$ & 6.6 & 0.51 & 170 & 0.15 & 0.92 & 210 & 0.17 & $\mathrm{~N}$ \\
\hline 47 & Chamaeleon I & $\mathrm{J} 1107161-772306$ & 6.6 & -0.10 & 490 & 0.076 & -0.03 & 590 & 0.099 & $\mathrm{~N}$ \\
\hline 48 & Chamaeleon I & $\mathrm{J} 1107213-772211$ & 6.6 & -0.08 & 650 & 0.089 & -0.45 & 820 & 0.13 & $\mathrm{~N}$ \\
\hline 49 & Chamaeleon I & J1107435-773941 & 5.9 & -1.05 & 1400 & 0.45 & -1.43 & 1700 & 0.85 & $\mathrm{~N}$ \\
\hline 50 & Chamaeleon I & $\mathrm{J} 1108029-773842$ & 6.6 & -0.10 & 710 & 0.97 & -0.39 & 900 & 1.4 & $\mathrm{~N}$ \\
\hline 51 & Chamaeleon I & J1109285-763328 & 6.6 & 1.17 & 260 & 0.90 & 1.30 & 300 & 1.1 & $\mathrm{Y}$ \\
\hline 52 & Chamaeleon I & J1109461-763446 & 5.9 & -0.41 & 720 & 0.18 & -1.13 & 1100 & 0.26 & $\mathrm{~N}$ \\
\hline 53 & Chamaeleon I & J1109472-772629 & 9.2 & -0.81 & 1100 & 0.092 & -1.25 & 1500 & 0.21 & $\mathrm{~N}$ \\
\hline 54 & Chamaeleon I & $\mathrm{J} 1110033-763311$ & 6.6 & 0.32 & 270 & 0.018 & -0.18 & 430 & 0.022 & $\mathrm{~N}$ \\
\hline 55 & Chamaeleon I & $\mathrm{J} 1110113-763529$ & 8.0 & -0.44 & 1100 & 0.37 & -1.24 & 1600 & 0.80 & $\mathrm{~N}$ \\
\hline 56 & Chamaeleon I & J1111107-764157 & 6.6 & 0.09 & 330 & 0.0076 & -0.20 & 650 & 0.0095 & $\mathrm{~N}$ \\
\hline 57 & Chamaeleon II & $\mathrm{J} 1253172-770710$ & 10.5 & -0.72 & 660 & 30 & -0.63 & 1500 & 63 & $\mathrm{~N}$ \\
\hline 58 & Chamaeleon II & $\mathrm{J} 1253428-771511$ & 4.0 & 0.65 & 130 & 0.43 & 0.32 & 160 & 0.45 & $\mathrm{Y}$ \\
\hline 59 & Chamaeleon II & J1259065-770739 & 4.0 & 0.68 & 230 & 1.7 & 1.12 & 260 & 1.8 & $\mathrm{Y}$ \\
\hline 60 & Corona Australis & $\mathrm{J} 1901480-365722$ & 7.9 & 0.78 & 93 & 4.4 & 1.03 & 130 & 4.7 & $\mathrm{Y}$ \\
\hline 61 & Corona Australis & $\mathrm{J} 1901484-365714$ & 7.9 & 1.41 & 17 & 3.7 & 1.20 & 23 & 3.7 & $\mathrm{Y}$ \\
\hline 62 & Corona Australis & J1901537-370033 & 1.5 & -1.09 & 19 & 1.4 & -1.29 & 23 & 1.4 & $\mathrm{Y}$ \\
\hline 63 & Corona Australis & $\mathrm{J} 1901585-365708$ & 7.9 & 0.88 & 13 & 6.9 & 0.72 & 15 & 7.0 & $\mathrm{Y}$ \\
\hline
\end{tabular}


Table 2

(Continued)

\begin{tabular}{|c|c|c|c|c|c|c|c|c|c|c|}
\hline \multirow[t]{2}{*}{ Index } & \multirow[t]{2}{*}{ Cloud } & \multirow{2}{*}{$\begin{array}{c}\text { Spitzer } \\
\text { Source Name } \\
(\mathrm{SSTc} 2 \mathrm{~d} \text { or SSTgb }+)\end{array}$} & \multirow[t]{2}{*}{$A_{V}{ }^{\mathrm{a}}$} & \multicolumn{3}{|c|}{ Observed } & \multicolumn{3}{|c|}{ Extinction Corrected } & \multirow[t]{2}{*}{ FIR/SMM ${ }^{\mathrm{b}}$} \\
\hline & & & & $\alpha$ & $\begin{array}{l}T_{\text {bol }} \\
(\mathrm{K})\end{array}$ & $\begin{array}{c}L_{\mathrm{bol}} \\
\left(L_{\odot}\right)\end{array}$ & $\alpha^{\prime}$ & $\begin{array}{c}T_{\mathrm{bol}}{ }^{\prime} \\
(\mathrm{K})\end{array}$ & $\begin{array}{l}L_{\mathrm{bol}}^{\prime} \\
\left(L_{\odot}\right)\end{array}$ & \\
\hline 64 & Corona Australis & J1902586-370735 & 7.9 & 1.66 & 61 & 1.2 & 1.48 & 66 & 1.3 & $\mathrm{Y}$ \\
\hline 65 & Corona Australis & J1901086-365720 & 7.9 & -0.80 & 1000 & 3.5 & -1.22 & 1500 & 6.9 & $\mathrm{Y}$ \\
\hline 66 & Corona Australis & J1903068-371249 & 7.9 & 0.36 & 460 & 8.8 & -0.21 & 610 & 13 & $\mathrm{Y}$ \\
\hline 67 & Corona Australis & J1901506-365809 & 7.9 & 0.92 & 210 & 15 & 1.01 & 270 & 17 & $\mathrm{Y}$ \\
\hline 68 & Corona Australis & J1901415-365831 & 7.9 & 0.75 & 270 & 7.1 & 0.27 & 390 & 8.5 & $\mathrm{Y}$ \\
\hline 69 & Corona Australis & J1901553-365721 & 7.9 & 2.64 & 260 & 0.44 & 2.47 & 310 & 0.53 & $\mathrm{Y}$ \\
\hline 70 & Corona Australis & J1901564-365728 & 7.9 & 2.78 & 200 & 1.4 & 2.56 & 210 & 1.8 & $\mathrm{Y}$ \\
\hline 71 & IC5146 & $\mathrm{J} 2145585+473601$ & 3.6 & 0.82 & 150 & 7.4 & 1.11 & 170 & 7.8 & $\mathrm{Y}$ \\
\hline 72 & IC5146 & $\mathrm{J} 2147227+473214$ & 3.6 & 0.74 & 86 & 35 & 0.63 & 90 & 36 & $\mathrm{Y}$ \\
\hline 73 & Lupus & J1539277-344617 & 1.0 & -0.84 & 2700 & 0.94 & -1.20 & 3300 & 1.3 & $\mathrm{~N}$ \\
\hline 74 & Lupus & J1539282-344618 & 2.0 & -0.84 & 2100 & 0.24 & -1.11 & 2600 & 0.36 & $\mathrm{~N}$ \\
\hline 75 & Lupus & J1607100-391103 & 3.0 & -1.04 & 2100 & 0.69 & -1.23 & 3700 & 1.6 & $\mathrm{~N}$ \\
\hline 76 & Lupus & J1608217-390421 & 1.0 & -1.13 & 2600 & 0.21 & -1.46 & 3100 & 0.28 & $\mathrm{~N}$ \\
\hline 77 & Lupus & J1608224-390446 & 0.0 & -0.48 & 1900 & 1.7 & -0.63 & 1900 & 1.7 & $\mathrm{~N}$ \\
\hline 78 & Lupus & J1609180-390453 & 2.9 & 1.10 & 39 & 0.41 & 1.14 & 39 & 0.41 & $\mathrm{Y}$ \\
\hline 79 & Ophiuchus & J1625381-242236 & 13.2 & -0.79 & 1100 & 0.11 & -1.22 & 1600 & 0.36 & $\mathrm{~N}$ \\
\hline 80 & Ophiuchus & J1625561-242048 & 4.5 & -0.60 & 1100 & 0.89 & -0.88 & 1400 & 1.3 & $\mathrm{~N}$ \\
\hline 81 & Ophiuchus & J1626103-242054 & 27.0 & -0.46 & 290 & 1.0 & -1.43 & 1300 & 3.7 & $\mathrm{Y}$ \\
\hline 82 & Ophiuchus & J1626146-242507 & 0.0 & $\ldots$ & 7 & 0.034 & $\ldots$ & 7 & 0.034 & $\mathrm{~N}$ \\
\hline 83 & Ophiuchus & J1626188-242819 & 19.7 & -0.73 & 990 & 0.50 & -1.28 & 1600 & 2.5 & $\mathrm{~N}$ \\
\hline 84 & Ophiuchus & $\mathrm{J} 1626213-242304$ & 9.8 & 1.46 & 210 & 8.6 & 1.14 & 250 & 11 & $\mathrm{Y}$ \\
\hline 85 & Ophiuchus & J1626236-244314 & 4.0 & -1.12 & 1500 & 0.48 & -1.34 & 1700 & 0.73 & $\mathrm{Y}$ \\
\hline 86 & Ophiuchus & $\mathrm{J} 1626240-241613$ & 13.3 & -0.71 & 980 & 1.9 & -1.09 & 1500 & 5.5 & $\mathrm{~N}$ \\
\hline 87 & Ophiuchus & $\mathrm{J} 1626254-242301$ & 9.8 & 0.87 & 140 & 0.010 & 0.60 & 200 & 0.12 & $\mathrm{~N}$ \\
\hline 88 & Ophiuchus & J1626256-242428 & 9.8 & 1.65 & 72 & 0.038 & 1.44 & 84 & 0.043 & $\mathrm{~N}$ \\
\hline 89 & Ophiuchus & J1626404-242714 & 9.8 & 0.45 & 380 & 0.064 & 0.32 & 470 & 0.092 & $\mathrm{~N}$ \\
\hline 90 & Ophiuchus & $\mathrm{J} 1626441-243448$ & 9.8 & 2.49 & 330 & 0.98 & 2.50 & 380 & 1.4 & $\mathrm{~N}$ \\
\hline 91 & Ophiuchus & $\mathrm{J} 1626450-242307$ & 18.5 & -0.64 & 820 & 0.29 & -1.20 & 1500 & 1.2 & $\mathrm{~N}$ \\
\hline 92 & Ophiuchus & J1626484-242838 & 9.8 & 0.02 & 440 & 0.12 & -0.06 & 570 & 0.17 & $\mathrm{~N}$ \\
\hline 93 & Ophiuchus & $\mathrm{J} 1626584-244531$ & 10.7 & -0.45 & 840 & 1.3 & -0.70 & 1300 & 2.7 & $\mathrm{~N}$ \\
\hline 94 & Ophiuchus & J1627023-243727 & 9.8 & 1.53 & 420 & 3.3 & 0.91 & 520 & 4.8 & $\mathrm{~N}$ \\
\hline 95 & Ophiuchus & J1627029-242614 & 9.8 & -0.19 & 380 & 0.036 & -0.23 & 550 & 0.050 & $\mathrm{~N}$ \\
\hline 96 & Ophiuchus & J1627052-243629 & 9.8 & 1.27 & 97 & 0.16 & 1.07 & 120 & 0.18 & $\mathrm{~N}$ \\
\hline 97 & Ophiuchus & J1627067-243814 & 9.8 & 0.61 & 330 & 0.48 & 0.73 & 420 & 0.64 & $\mathrm{~N}$ \\
\hline 98 & Ophiuchus & $\mathrm{J} 1627094-243718$ & 9.8 & 1.69 & 370 & 13 & 1.06 & 420 & 18 & $\mathrm{~N}$ \\
\hline 99 & Ophiuchus & $\mathrm{J} 1627158-243843$ & 18.6 & -0.70 & 220 & 0.63 & -1.64 & 830 & 1.1 & $\mathrm{~N}$ \\
\hline 100 & Ophiuchus & J1627175-242856 & 9.8 & 0.25 & 190 & 0.52 & 0.37 & 260 & 0.61 & $\mathrm{~N}$ \\
\hline 101 & Ophiuchus & J1627214-244143 & 9.8 & -0.03 & 610 & 1.1 & -0.02 & 720 & 1.8 & $\mathrm{~N}$ \\
\hline 102 & Ophiuchus & $\mathrm{J} 1627218-242727$ & 9.8 & -0.05 & 180 & 0.019 & -0.08 & 290 & 0.022 & $\mathrm{~N}$ \\
\hline 103 & Ophiuchus & $\mathrm{J} 1627245-244103$ & 9.8 & 1.01 & 170 & 0.31 & 1.23 & 230 & 0.37 & $\mathrm{~N}$ \\
\hline 104 & Ophiuchus & J1627269-244050 & 9.8 & 1.17 & 240 & 2.6 & 1.15 & 300 & 3.3 & $\mathrm{~N}$ \\
\hline 105 & Ophiuchus & $\mathrm{J} 1627279-243933$ & 9.8 & 2.29 & 260 & 5.0 & 2.13 & 280 & 7.1 & $\mathrm{~N}$ \\
\hline 106 & Ophiuchus & J1627284-242721 & 9.8 & -0.03 & 310 & 0.48 & -0.13 & 450 & 0.63 & $\mathrm{~N}$ \\
\hline 107 & Ophiuchus & $\mathrm{J} 1627301-242743$ & 9.8 & -0.12 & 500 & 0.97 & -0.02 & 620 & 1.5 & $\mathrm{~N}$ \\
\hline 108 & Ophiuchus & J1627372-244237 & 9.8 & 0.13 & 460 & 0.12 & 0.15 & 560 & 0.18 & $\mathrm{~N}$ \\
\hline 109 & Ophiuchus & J1627398-244315 & 9.8 & -0.15 & 570 & 0.72 & -0.15 & 690 & 1.1 & $\mathrm{~N}$ \\
\hline 110 & Ophiuchus & $\mathrm{J} 1628216-243623$ & 9.8 & 1.23 & 33 & 0.24 & 0.96 & 36 & 0.24 & $\mathrm{Y}$ \\
\hline 111 & Ophiuchus & $\mathrm{J} 1628578-244054$ & 9.8 & 0.67 & 320 & 0.027 & 0.58 & 430 & 0.037 & $\mathrm{~N}$ \\
\hline 112 & Ophiuchus & J1631356-240129 & 9.8 & 0.14 & 270 & 1.6 & 0.14 & 390 & 2.0 & $\mathrm{Y}$ \\
\hline 113 & Ophiuchus & J1631367-240419 & 9.8 & -0.27 & 74 & 0.17 & -0.22 & 160 & 0.19 & $\mathrm{Y}$ \\
\hline 114 & Ophiuchus & J1631437-245524 & 9.8 & 0.23 & 520 & 0.26 & 0.19 & 690 & 0.40 & $\mathrm{~N}$ \\
\hline 115 & Ophiuchus & J1631520-245726 & 9.8 & 0.82 & 120 & 0.0082 & 0.61 & 150 & 0.0095 & $\mathrm{~N}$ \\
\hline 116 & Ophiuchus & J1631524-245536 & 9.8 & 1.07 & 260 & 0.11 & 0.87 & 330 & 0.15 & $\mathrm{~N}$ \\
\hline 117 & Ophiuchus & $\mathrm{J} 1632009-245642$ & 9.8 & 1.39 & 140 & 2.5 & 1.36 & 180 & 2.8 & $\mathrm{Y}$ \\
\hline 118 & Ophiuchus & J1632226-242831 & 9.8 & 5.03 & 45 & 8.5 & 4.87 & 45 & 8.8 & $\mathrm{Y}$ \\
\hline 119 & Ophiuchus & J1633556-244205 & 4.6 & -1.22 & 1500 & 0.17 & -1.31 & 1800 & 0.29 & $\mathrm{~N}$ \\
\hline 120 & Ophiuchus North & $\mathrm{J} 1646582-093519$ & 5.6 & 0.66 & 230 & 0.44 & 0.61 & 280 & 0.50 & $\mathrm{Y}$ \\
\hline 121 & Ophiuchus North & $\mathrm{J} 1648456-141636$ & 5.6 & -0.97 & 1400 & 1.2 & -1.39 & 1700 & 2.2 & $\mathrm{~N}$ \\
\hline 122 & Ophiuchus North & J1657196-160923 & 5.7 & 2.40 & 38 & 0.80 & 2.57 & 39 & 0.82 & $\mathrm{Y}$ \\
\hline 123 & Perseus & $\mathrm{J} 0325223+304513$ & 5.9 & 2.34 & 52 & 2.0 & 2.20 & 53 & 2.1 & $\mathrm{Y}$ \\
\hline 124 & Perseus & J0325362+304515 & 5.9 & 1.59 & 12 & 8.5 & 1.49 & 12 & 8.5 & $\mathrm{Y}$ \\
\hline 125 & Perseus & J0325364+304522 & 5.9 & 2.62 & 66 & 4.9 & 2.55 & 70 & 5.2 & $\mathrm{Y}$ \\
\hline 126 & Perseus & J0325388+304406 & 5.9 & 2.16 & 47 & 6.9 & 2.04 & 48 & 7.0 & $\mathrm{Y}$ \\
\hline
\end{tabular}


Table 2

(Continued)

\begin{tabular}{|c|c|c|c|c|c|c|c|c|c|c|}
\hline \multirow[t]{2}{*}{ Index } & \multirow[t]{2}{*}{ Cloud } & \multirow{2}{*}{$\begin{array}{c}\text { Spitzer } \\
\text { Source Name } \\
(\text { SSTc2d or SSTgb +) }\end{array}$} & \multirow[t]{2}{*}{$A_{V}{ }^{\mathrm{a}}$} & \multicolumn{3}{|c|}{ Observed } & \multicolumn{3}{|c|}{ Extinction Corrected } & \multirow[t]{2}{*}{ FIR/SMM ${ }^{\mathrm{b}}$} \\
\hline & & & & $\alpha$ & $\begin{array}{c}T_{\text {bol }} \\
(\mathrm{K})\end{array}$ & $\begin{array}{c}L_{\text {bol }} \\
\left(L_{\odot}\right)\end{array}$ & $\alpha^{\prime}$ & $\begin{array}{c}T_{\text {bol }}{ }^{\prime} \\
(\mathrm{K})\end{array}$ & $\begin{array}{l}L_{\mathrm{boll}}^{\prime} \\
\left(L_{\odot}\right)\end{array}$ & \\
\hline 127 & Perseus & $\mathrm{J} 0325391+304358$ & 5.9 & 2.36 & 160 & 0.69 & 2.02 & 170 & 0.78 & $\mathrm{~N}$ \\
\hline 128 & Perseus & J0326374+301528 & 5.9 & 1.09 & 64 & 0.91 & 1.02 & 73 & 0.94 & $\mathrm{Y}$ \\
\hline 129 & Perseus & $\mathrm{J} 0327382+301358$ & 5.9 & -0.19 & 260 & 0.78 & -0.40 & 360 & 0.89 & $\mathrm{Y}$ \\
\hline 130 & Perseus & $\mathrm{J} 0327390+301303$ & 5.9 & 2.68 & 62 & 3.5 & 2.45 & 65 & 3.6 & $\mathrm{Y}$ \\
\hline 131 & Perseus & J0327432+301228 & 5.9 & 2.39 & 54 & 1.7 & 2.23 & 57 & 1.7 & $\mathrm{Y}$ \\
\hline 132 & Perseus & J0327476+301204 & 5.9 & -0.09 & 740 & 2.5 & -0.25 & 950 & 3.6 & $\mathrm{Y}$ \\
\hline 133 & Perseus & $\mathrm{J} 0328003+300801$ & 5.9 & 0.95 & 230 & 0.25 & 0.99 & 280 & 0.29 & $\mathrm{Y}$ \\
\hline 134 & Perseus & $\mathrm{J} 0328325+311105$ & 5.9 & 0.78 & 52 & 0.26 & 0.50 & 74 & 0.27 & $\mathrm{Y}$ \\
\hline 135 & Perseus & J0328344+310051 & 5.9 & 0.83 & 240 & 1.1 & 0.88 & 290 & 1.3 & $\mathrm{Y}$ \\
\hline 136 & Perseus & J0328345+310705 & 5.9 & 0.54 & 150 & 0.12 & 0.32 & 170 & 0.13 & $\mathrm{Y}$ \\
\hline 137 & Perseus & $\mathrm{J} 0328350+302009$ & 5.9 & 0.15 & 49 & 0.36 & -0.22 & 89 & 0.36 & $\mathrm{Y}$ \\
\hline 138 & Perseus & $\mathrm{J} 0328370+311330$ & 5.9 & 2.35 & 100 & 9.5 & 1.94 & 110 & 10 & $\mathrm{Y}$ \\
\hline 139 & Perseus & J0328391+310601 & 5.9 & 1.68 & 28 & 0.23 & 1.55 & 29 & 0.23 & $\mathrm{Y}$ \\
\hline 140 & Perseus & J0328397+311731 & 5.9 & 0.57 & 250 & 0.18 & 0.61 & 300 & 0.21 & $\mathrm{Y}$ \\
\hline 141 & Perseus & J0328406+311756 & 5.9 & 1.02 & 12 & 0.58 & 0.95 & 12 & 0.58 & $\mathrm{Y}$ \\
\hline 142 & Perseus & J0328432+311732 & 5.9 & 0.36 & 490 & 1.6 & 0.25 & 640 & 2.0 & $\mathrm{Y}$ \\
\hline 143 & Perseus & J0328453+310541 & 5.9 & 1.11 & 62 & 0.41 & 1.08 & 72 & 0.43 & $\mathrm{Y}$ \\
\hline 144 & Perseus & J0328555+311436 & 5.9 & 3.03 & 54 & 22 & 2.37 & 55 & 22 & $\mathrm{Y}$ \\
\hline 145 & Perseus & J0328563+312227 & 5.9 & -0.14 & 440 & 0.15 & -0.40 & 620 & 0.19 & $\mathrm{~N}$ \\
\hline 146 & Perseus & $\mathrm{J} 0328573+311415$ & 5.9 & 1.60 & 100 & 5.3 & 1.40 & 110 & 5.7 & $\mathrm{Y}$ \\
\hline 147 & Perseus & J0328584+312217 & 5.9 & 0.83 & 240 & 0.96 & 0.83 & 280 & 1.1 & $\mathrm{Y}$ \\
\hline 148 & Perseus & J0328593+311548 & 5.9 & 0.06 & 10 & 3.2 & -0.08 & 140 & 3.4 & $\mathrm{Y}$ \\
\hline 149 & Perseus & $\mathrm{J} 0329005+311200$ & 5.9 & 2.16 & 30 & 0.66 & 1.96 & 30 & 0.67 & $\mathrm{Y}$ \\
\hline 150 & Perseus & J0329015+312020 & 5.9 & 2.09 & 230 & 8.2 & 2.30 & 270 & 9.4 & $\mathrm{Y}$ \\
\hline 151 & Perseus & J0329033+312314 & 5.9 & 1.13 & 320 & 0.088 & 1.01 & 370 & 0.11 & $\mathrm{~N}$ \\
\hline 152 & Perseus & J0329037+311603 & 5.9 & 1.21 & 170 & 33 & 0.85 & 220 & 37 & Y \\
\hline 153 & Perseus & J0329040+311446 & 5.9 & 1.43 & 17 & 0.68 & 1.31 & 19 & 0.68 & $\mathrm{Y}$ \\
\hline 154 & Perseus & J0329077+312157 & 5.9 & 2.18 & 230 & 18 & 2.31 & 260 & 21 & $\mathrm{Y}$ \\
\hline 155 & Perseus & J0329104+311331 & 5.9 & 2.58 & 31 & 7.9 & 2.48 & 31 & 8.0 & $\mathrm{Y}$ \\
\hline 156 & Perseus & J0329106+311820 & 5.9 & 1.95 & 56 & 3.2 & 1.84 & 58 & 3.3 & $\mathrm{Y}$ \\
\hline 157 & Perseus & J0329112+311831 & 5.9 & 1.94 & 29 & 1.2 & 1.77 & 32 & 1.3 & $\mathrm{Y}$ \\
\hline 158 & Perseus & J0329120+311305 & 5.9 & 0.98 & 25 & 4.2 & 0.81 & 25 & 4.2 & $\mathrm{Y}$ \\
\hline 159 & Perseus & J0329129+311814 & 5.9 & 1.05 & 240 & 0.100 & 1.58 & 270 & 1.1 & $\mathrm{~N}$ \\
\hline 160 & Perseus & J0329135+311358 & 5.9 & 2.41 & 35 & 0.84 & 2.50 & 36 & 0.85 & $\mathrm{Y}$ \\
\hline 161 & Perseus & J0329171+312746 & 5.9 & 1.75 & 32 & 0.65 & 1.70 & 34 & 0.66 & $\mathrm{Y}$ \\
\hline 162 & Perseus & J0329182+312319 & 5.9 & 1.26 & 21 & 0.54 & 1.11 & 24 & 0.54 & $\mathrm{Y}$ \\
\hline 163 & Perseus & J0329187+312325 & 5.9 & -0.23 & 190 & 1.9 & -0.67 & 410 & 2.1 & $\mathrm{Y}$ \\
\hline 164 & Perseus & J0329200+312407 & 5.9 & 0.42 & 75 & 1.5 & 0.34 & 10 & 1.6 & $\mathrm{Y}$ \\
\hline 165 & Perseus & J0329234+313329 & 5.9 & 1.51 & 60 & 0.36 & 1.51 & 64 & 0.37 & $\mathrm{Y}$ \\
\hline 166 & Perseus & J0329518+313906 & 5.9 & 3.44 & 39 & 0.50 & 3.40 & 40 & 0.51 & $\mathrm{Y}$ \\
\hline 167 & Perseus & $\mathrm{J} 0330151+302349$ & 5.9 & 1.70 & 93 & 1.4 & 1.57 & 100 & 1.5 & $\mathrm{Y}$ \\
\hline 168 & Perseus & $\mathrm{J} 0330326+302626$ & 5.9 & 2.08 & 34 & 0.16 & 2.72 & 35 & 0.16 & $\mathrm{Y}$ \\
\hline 169 & Perseus & J0331209+304530 & 5.9 & 0.98 & 32 & 1.2 & 1.48 & 32 & 1.2 & $\mathrm{Y}$ \\
\hline 170 & Perseus & J0332179+304947 & 5.9 & 1.07 & 25 & 1.3 & 0.87 & 26 & 1.3 & $\mathrm{Y}$ \\
\hline 171 & Perseus & $\mathrm{J} 0332291+310240$ & 5.9 & 0.40 & 120 & 0.52 & 0.16 & 160 & 0.56 & $\mathrm{Y}$ \\
\hline 172 & Perseus & J0333095+310531 & 5.9 & 1.13 & 210 & 0.042 & 0.69 & 320 & 0.049 & $\mathrm{~N}$ \\
\hline 173 & Perseus & J0333128+312124 & 5.9 & 0.41 & 480 & 2.9 & 0.16 & 610 & 3.8 & $\mathrm{Y}$ \\
\hline 174 & Perseus & J0333138+312005 & 5.9 & 1.44 & 59 & 0.100 & 1.31 & 69 & 0.11 & $\mathrm{Y}$ \\
\hline 175 & Perseus & $\mathrm{J} 0333143+310710$ & 5.9 & 2.22 & 34 & 0.63 & 2.73 & 36 & 0.64 & $\mathrm{Y}$ \\
\hline 176 & Perseus & J0333164+310652 & 5.9 & 1.73 & 26 & 1.0 & 1.56 & 26 & 1.0 & $\mathrm{Y}$ \\
\hline 177 & Perseus & J0333166+310755 & 5.9 & 1.57 & 100 & 1.6 & 1.69 & 120 & 1.7 & $\mathrm{Y}$ \\
\hline 178 & Perseus & J0333178+310931 & 5.9 & 3.33 & 41 & 3.5 & 2.92 & 44 & 3.6 & $\mathrm{Y}$ \\
\hline 179 & Perseus & J0333203+310721 & 5.9 & 0.88 & 47 & 0.60 & 0.61 & 55 & 0.61 & $\mathrm{Y}$ \\
\hline 180 & Perseus & $\mathrm{J} 0333272+310710$ & 5.9 & 1.93 & 62 & 1.3 & 1.79 & 66 & 1.4 & $\mathrm{Y}$ \\
\hline 181 & Perseus & J0342021+314802 & 5.9 & 1.47 & 190 & 0.069 & 1.23 & 240 & 0.080 & $\mathrm{~N}$ \\
\hline 182 & Perseus & $\mathrm{J} 0343451+320358$ & 5.9 & -0.22 & 540 & 0.71 & 0.12 & 650 & 0.93 & $\mathrm{~N}$ \\
\hline 183 & Perseus & $\mathrm{J} 0343509+320324$ & 5.9 & 1.51 & 16 & 0.68 & 1.38 & 17 & 0.69 & $\mathrm{Y}$ \\
\hline 184 & Perseus & $\mathrm{J} 0343510+320308$ & 5.9 & -0.28 & 52 & 0.40 & -0.30 & 57 & 0.41 & $\mathrm{Y}$ \\
\hline 185 & Perseus & J0343565+320052 & 5.9 & 0.50 & 23 & 1.6 & 0.55 & 23 & 1.6 & $\mathrm{Y}$ \\
\hline 186 & Perseus & J0343568+320304 & 5.9 & 1.37 & 22 & 1.4 & 1.83 & 23 & 1.4 & $\mathrm{Y}$ \\
\hline 187 & Perseus & J0343596+320154 & 19.8 & -0.34 & 620 & 1.8 & -0.90 & 1300 & 6.2 & $\mathrm{Y}$ \\
\hline 188 & Perseus & J0344024+320204 & 5.9 & 1.53 & 41 & 0.35 & 1.47 & 45 & 0.35 & $\mathrm{Y}$ \\
\hline 189 & Perseus & $\mathrm{J} 0344129+320135$ & 5.9 & 0.37 & 400 & 1.6 & 0.30 & 470 & 2.0 & $\mathrm{~N}$ \\
\hline
\end{tabular}


Table 2

(Continued)

\begin{tabular}{|c|c|c|c|c|c|c|c|c|c|c|}
\hline \multirow[t]{2}{*}{ Index } & \multirow[t]{2}{*}{ Cloud } & \multirow{2}{*}{$\begin{array}{c}\text { Spitzer } \\
\text { Source Name } \\
(\text { SSTc2d or SSTgb +) }\end{array}$} & \multirow[t]{2}{*}{$A_{V}{ }^{\mathrm{a}}$} & \multicolumn{3}{|c|}{ Observed } & \multicolumn{3}{|c|}{ Extinction Corrected } & \multirow[t]{2}{*}{ FIR/SMM ${ }^{\mathrm{b}}$} \\
\hline & & & & $\alpha$ & $\begin{array}{c}T_{\text {bol }} \\
(\mathrm{K})\end{array}$ & $\begin{array}{c}L_{\mathrm{bol}} \\
\left(L_{\odot}\right)\end{array}$ & $\alpha^{\prime}$ & $\begin{array}{c}T_{\mathrm{bol}}{ }^{\prime} \\
(\mathrm{K})\end{array}$ & $\begin{array}{l}L_{\mathrm{bol}}{ }^{\prime} \\
\left(L_{\odot}\right)\end{array}$ & \\
\hline 191 & Perseus & J0344433+320131 & 5.9 & 0.50 & 460 & 1.2 & 0.62 & 500 & 1.5 & $\mathrm{~N}$ \\
\hline 192 & Perseus & J0344439+320136 & 5.9 & 0.96 & 39 & 3.1 & 0.73 & 41 & 3.1 & $\mathrm{Y}$ \\
\hline 193 & Perseus & J0347054+324308 & 5.9 & 0.36 & 330 & 0.49 & 0.52 & 390 & 0.58 & $\mathrm{Y}$ \\
\hline 194 & Perseus & $\mathrm{J} 0347415+325144$ & 5.9 & 0.78 & 290 & 4.4 & 1.33 & 330 & 5.1 & $\mathrm{Y}$ \\
\hline 197 & Serpens & $\mathrm{J} 1828449+005203$ & 9.6 & 1.33 & 53 & 3.9 & 1.12 & 62 & 4.1 & $\mathrm{Y}$ \\
\hline 198 & Serpens & $\mathrm{J} 1828512+001927$ & 9.6 & 0.45 & 260 & 0.14 & 0.28 & 390 & 0.18 & $\mathrm{~N}$ \\
\hline 199 & Serpens & $\mathrm{J} 1828540+002930$ & 9.6 & 1.36 & 60 & 8.3 & 1.14 & 69 & 8.8 & $\mathrm{Y}$ \\
\hline 200 & Serpens & $\mathrm{J} 1828548+002952$ & 9.6 & 1.91 & 47 & 6.3 & 1.60 & 51 & 6.6 & $\mathrm{Y}$ \\
\hline 201 & Serpens & $\mathrm{J} 1828549+001832$ & 9.6 & 0.90 & 120 & 0.12 & 0.68 & 150 & 0.14 & $\mathrm{~N}$ \\
\hline 202 & Serpens & $\mathrm{J} 1828557+002944$ & 9.6 & 1.89 & 22 & 1.5 & 1.65 & 26 & 1.6 & $\mathrm{Y}$ \\
\hline 207 & Serpens & $\mathrm{J} 1829090+003132$ & 9.6 & 2.27 & 35 & 4.1 & 2.12 & 36 & 4.2 & $\mathrm{Y}$ \\
\hline 208 & Serpens & $\mathrm{J} 1829161+001822$ & 9.6 & -0.07 & 440 & 4.6 & 0.07 & 620 & 6.7 & $\mathrm{Y}$ \\
\hline 209 & Serpens & $\mathrm{J} 1829319+011842$ & 9.6 & 0.26 & 450 & 14 & -0.06 & 710 & 21 & $\mathrm{Y}$ \\
\hline 210 & Serpens & $\mathrm{J} 1829481+011644$ & 9.6 & 1.37 & 29 & 14 & 1.11 & 30 & 14 & $\mathrm{Y}$ \\
\hline 211 & Serpens & J1829491+011619 & 9.6 & 3.80 & 130 & 11 & 3.45 & 150 & 13 & $\mathrm{~N}$ \\
\hline 212 & Serpens & J1829496+011521 & 9.6 & 2.65 & 13 & 69 & 2.53 & 13 & 69 & $\mathrm{Y}$ \\
\hline 213 & Serpens & $\mathrm{J} 1829511+011640$ & 9.6 & 1.04 & 130 & 4.8 & 1.07 & 170 & 5.4 & $\mathrm{Y}$ \\
\hline 214 & Serpens & $\mathrm{J} 1829522+011547$ & 9.6 & 1.54 & 62 & 7.6 & 1.26 & 69 & 8.1 & $\mathrm{Y}$ \\
\hline 215 & Serpens & $\mathrm{J} 1829525+003611$ & 9.6 & 0.77 & 51 & 1.8 & 0.53 & 68 & 1.9 & $\mathrm{Y}$ \\
\hline 216 & Serpens & J1829528+011456 & 9.6 & 1.49 & 120 & 3.0 & 1.19 & 140 & 3.4 & $\mathrm{~N}$ \\
\hline 217 & Serpens & $\mathrm{J} 1829543+003601$ & 9.6 & -0.18 & 42 & 1.7 & -0.34 & 59 & 1.7 & $\mathrm{Y}$ \\
\hline 218 & Serpens & $\mathrm{J} 1829568+011446$ & 9.6 & 0.30 & 330 & 16 & 0.33 & 500 & 21 & $\mathrm{~N}$ \\
\hline 219 & Serpens & $\mathrm{J} 1829575+011300$ & 9.6 & 1.01 & 42 & 28 & 1.14 & 59 & 29 & $\mathrm{Y}$ \\
\hline 228 & Serpens & $\mathrm{J} 1830027+011228$ & 9.6 & 0.21 & 390 & 5.5 & 0.19 & 540 & 7.7 & $\mathrm{~N}$ \\
\hline 229 & Serpens & $\mathrm{J} 1830052+004104$ & 9.6 & 1.30 & 95 & 0.19 & 1.04 & 100 & 0.21 & $\mathrm{~N}$ \\
\hline 230 & Serpens & $\mathrm{J} 1830057+003931$ & 30.0 & -0.39 & 540 & 0.18 & -1.18 & 1500 & 1.1 & $\mathrm{~N}$ \\
\hline
\end{tabular}

Notes.

a Value of $A_{V}$ used for dereddening, as explained in the text.

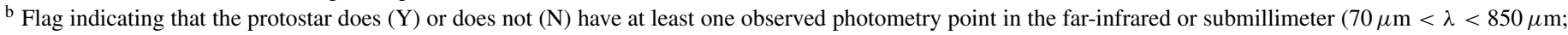
see Section 4.3 for details).

Statistical uncertainties calculated by propagating through the uncertainties in the observed fluxes are on the order of $10 \%$, but the true uncertainties are dominated by incomplete sampling of the SEDs and are impossible to calculate for each source individually. These uncertainties will be discussed further in Section 4.3.

Figure 1 shows the distribution of the extinction corrected values of $L_{\mathrm{bol}}$ for all 230 protostars in log space. With a minimum and maximum of $0.01 L_{\odot}$ and $69 L_{\odot}$, respectively, this distribution extends over greater than three orders of magnitude. The mean and median are $4.3 L_{\odot}$ and $1.3 L_{\odot}$, respectively. These statistics are summarized in Table 3. Also listed in Table 3 are four dimensionless quantities calculated from the luminosity distribution: the standard deviation of $\log$
$L_{\mathrm{bol}}$, the ratio of the median to mean $L_{\mathrm{bol}}$, the ratio of the maximum to mean $L_{\mathrm{bol}}$, and the fraction of protostars with $L_{\text {bol }} \leqslant 0.1 L_{\odot}$. These particular quantities are motivated by the recent theoretical study of protostellar luminosities by Offner $\&$ McKee (2011), to which we compare our results below in Section 4.2.

Figure 2 shows the $L_{\text {bol }}$ distributions separately for Class 0 and Class I sources. We have used $T_{\mathrm{bol}}$, calculated using Equation (3), to classify our sources, since $T_{\text {bol }}$ is one of several commonly used indicators of class and evolutionary status (e.g., Dunham et al. 2008; Enoch et al. 2009; Evans et al. 2009; Maury et al. 2011). Following Chen et al. (1995), Class 0 sources are selected with the criterion that $T_{\text {bol }}<70 \mathrm{~K}$ and Class I sources are selected with the criterion that $70 \leqslant T_{\text {bol }} \leqslant 650 \mathrm{~K}$. 


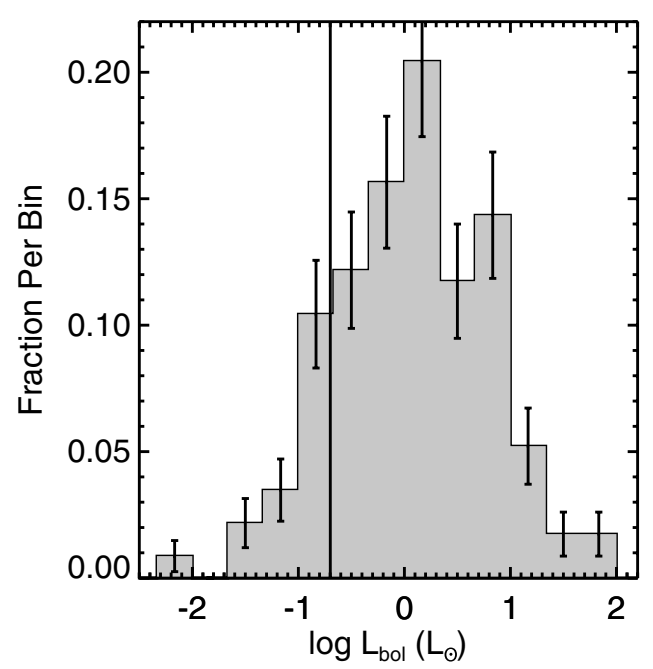

Figure 1. Histogram showing the distribution of extinction corrected $L_{\mathrm{bol}}$ for all 230 protostars in log space. The bins are $1 / 3$ dex wide, and the error bars show the statistical $(\sqrt{N})$ uncertainties. The solid vertical line shows the approximate completeness limit of $0.2 L_{\odot}$ for the c $2 \mathrm{~d}+\mathrm{GB}$ sample.

Table 3

Luminosity Distribution Statistics

\begin{tabular}{lc}
\hline \hline Parameter & Value \\
\hline Total number & 230 \\
Mean & $4.3^{\mathrm{a}} L_{\odot}$ \\
Median & $1.3^{\mathrm{a}} L_{\odot}$ \\
Minimum & $0.01 L_{\odot}$ \\
Maximum & $69 L_{\odot}$ \\
Standard deviation of log & 0.73 \\
Median/mean & 0.3 \\
Maximum/mean & 16.0 \\
Fraction $\leqslant 0.1 L_{\odot}$ & 0.07
\end{tabular}

Notes. ${ }^{\text {a }}$ As described in Section 4.3, once far-infrared and submillimeter photometry becomes available for the $43 \%$ of the sample lacking any available data at $70 \mu \mathrm{m}<\lambda<850 \mu \mathrm{m}$, the mean and median will likely increase to approximately 5.8 and $1.8 L_{\odot}$, respectively. The effects of including such data on the overall distribution of $L_{\mathrm{bol}}$, and thus on the other quantities listed in this table, can only be investigated once such data are available.

Inspection of Figure 2 reveals that the peak and extent of the $L_{\text {bol }}$ distributions are similar for Class 0 and Class I sources. The distributions have mean (median) values of $4.5 L_{\odot}$ and $3.8 L_{\odot}\left(1.4 L_{\odot}\right.$ and $\left.1.0 L_{\odot}\right)$ for the Class 0 and I sources, respectively. However, there is one significant difference in that there is an excess of low luminosity Class I sources compared to the Class 0 population. For the Class I population, $36 \%$ have $L_{\text {bol }}<0.5 L_{\odot}$, whereas for the Class 0 population, only $20 \%$ have such luminosities. A K-S test on the two distributions returns a value of only 0.04 , demonstrating that the difference at low luminosities is statistically significant. These results are similar to those obtained by Enoch et al. (2009) for a smaller sample. Very recently, several extremely low luminosity, Class 0 sources have been discovered in cores classified as starless based on Spitzer observations (e.g., Chen et al. 2010; Enoch et al. 2010; Pineda et al. 2011; Schnee et al. 2012; Chen et al. 2012), emphasizing that at least some of this difference may be due to a bias against the lowest luminosity Class 0 sources in Spitzer-selected samples. This point is further emphasized

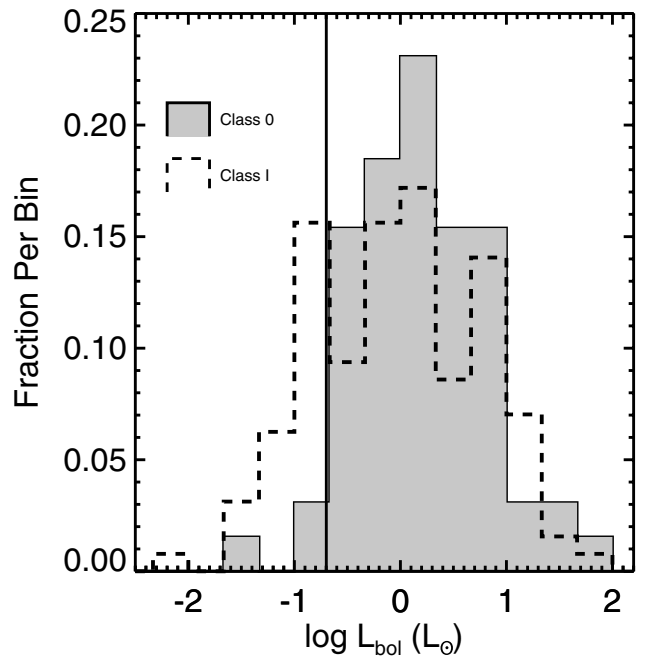

Figure 2. Histogram showing $L_{\text {bol }}$ distributions in log space with $1 / 3$ dex bins. The shaded histogram shows the distribution derived in this study for the 65 out of 230 objects in the combined c2d and GB samples classified as Class 0 protostars. The dashed histogram shows the same thing, except for the 120 out of 230 objects classified as Class I protostars. The classification is based on $T_{\text {bol }}$ calculated according to Equation 3 using the extinction-corrected photometry and the Class boundaries defined by Chen et al. (1995). The solid vertical line shows the approximate completeness limit of $0.2 L_{\odot}$ for the c $2 \mathrm{~d}+\mathrm{GB}$ sample.

Table 4

$L_{\text {bol }}$ and $L_{\text {int }}$ for VeLLOs

\begin{tabular}{lccc}
\hline \hline Source & $L_{\text {bol }}$ & $L_{\text {int }}$ & Reference $^{\mathrm{a}}$ \\
\hline L1014-IRS & 0.34 & 0.09 & 1 \\
IRAM04191-IRS & 0.13 & 0.08 & 2 \\
L1521F-IRS & 0.13 & 0.05 & 3 \\
L328-IRS & 0.18 & 0.05 & 4 \\
L673-7-IRS & 0.18 & 0.04 & 5 \\
L1148-IRS & 0.13 & $0.08-0.13$ & 6,7 \\
\hline
\end{tabular}

References. (1) Young et al. 2004; (2) Dunham et al. 2006; (3) Bourke et al. 2006; (4) Lee et al. 2009; (5) Dunham et al. 2010a; (6) Kauffmann et al. 2005; (7) Kauffmann et al. 2011.

by the fact that the excess of low-luminosity Class I sources occurs below our approximate completeness limit of $0.2 L_{\odot}$ (see below), where any such comparisons are limited in utility. The true similarity of the Class 0 and Class I $L_{\text {bol }}$ distributions must be revisited once current and future surveys with Herschel and ALMA detect and characterize the full population of extremely low luminosity protostars.

We emphasize that the results presented here are the observed bolometric luminosities of protostars, which are not the same as the intrinsic protostellar luminosities. Departures from spherical symmetry break the correlation between observed and intrinsic bolometric luminosities, and external heating from the interstellar radiation field breaks the correlation between bolometric and internal luminosity. Regarding the latter, external heating can add up to several tenths of a solar luminosity depending on the local strength of the interstellar radiation field and the core mass available to be heated externally (e.g., Evans et al. 2001) and can dominate the observed $L_{\text {bol }}$ for the lowest luminosity objects. A few specific examples of this point can be found in recent, detailed studies of individual VeLLOs that use continuum radiative transfer models to separate internal and external heating and determine the intrinsic $L_{\text {int }}$. The observed $L_{\text {bol }}$ and model-derived $L_{\text {int }}$ for six such sources are listed in Table 4 . For at least five and possibly all six, the observed $L_{\text {bol }}$ 
are above $0.1 L_{\odot}$ while the model-derived $L_{\text {int }}$ are below $0.1 L_{\odot}$, qualifying them as VeLLOs. As a consequence, the fraction of protostars with $L_{\text {bol }} \leqslant 0.1 L_{\odot}$ reported in Table $3(0.07)$ does not imply that $7 \%$ of the sample are VeLLOs; many more VeLLOs with $L_{\text {bol }}>0.1 L_{\odot}$ are likely present in the sample.

We have decided not to attempt to correct our luminosity distribution for source inclination or external heating, since any such corrections would be model-dependent (and in the case of external heating would require detailed modeling of all lowluminosity protostars, a project far beyond the scope of this paper). What we present are simply the observed bolometric luminosities (after correcting for extinction). Theoretical studies that attempt to explain the observed protostellar luminosity distribution must take these considerations into account.

Since the relationship between the fluxes in the various Spitzer bands and $L_{\text {bol }}$ depends not only on distance but also on the detailed spectral shape of each source, local strength of the external (interstellar) radiation field, and total core mass available to be heated externally, there is no one completeness limit for each cloud or for the full $\mathrm{c} 2 \mathrm{~d}+\mathrm{GB}$ data set. In a detailed search for and study of low luminosity protostars in the c2d survey, Dunham et al. (2008) found that the $\mathrm{c} 2 \mathrm{~d}$ data are sensitive to protostars with $L_{\text {int }} \geqslant 4 \times 10^{-3}(d / 140 \mathrm{pc})^{2} L_{\odot}$. With cloud distances ranging from $125-950 \mathrm{pc}$, the resulting luminosity sensitivities range from $0.003-0.18 L_{\odot}$, or $0.003-0.04 L_{\odot}$ if IC5146 is omitted. However, this sensitivity is for $L_{\text {int }}$ rather than $L_{\text {bol }}$; as discussed above, the two are not the same for low luminosity protostars, with $L_{\text {bol }}$ equal to or greater than $L_{\text {int }}$ depending on the details of the external heating. In another study, Enoch et al. (2009) estimated completeness limits of $L_{\text {bol }} \sim 0.01-0.05 L_{\odot}$ for protostars in the c2d clouds, although they emphasized that there was significant uncertainty in deriving such limits. Taking into account all of the above information, we conservatively estimate that our sample is only complete for $L_{\text {bol }}>0.2 L_{\odot}$ (the sensitivity limit for IC5146, the most distant cloud, using the Dunham et al. (2008) relation and assuming no external heating), and mark this limit with a solid vertical line in all figures presenting histograms of $L_{\mathrm{bol}}$. The existence of protostars below these limits will be discussed in Section 5.3.

\section{DISCUSSION}

In this section we discuss our results in comparison to other observational and theoretical studies of protostellar luminosities. In Section 4.1 we compare to two recent determinations of the observed protostellar luminosity distribution, and in Section 4.2 we discuss several recent theoretical investigations of protostellar luminosities. Finally, in Section 4.3 we discuss the effects of missing far-infrared and submillimeter photometry on our derived luminosities.

\subsection{Comparison to Other Observations}

\subsubsection{Comparison to c2d Results}

Evans et al. (2009) identified 112 protostars in the c2d survey and calculated their observed bolometric luminosities. Our methods for identifying protostars, assembling complete SEDs, and calculating $L_{\text {bol }}$ are very similar to theirs. All of their protostars are included in our study, but we have expanded to the rest of the star-forming clouds observed by the GB survey and thus increased the number of protostars from 112 to 230 . We have also made three changes to the ancillary photometry included when assembling complete SEDs: (1) we have included 12 and $22 \mu \mathrm{m}$ photometry from the WISE all-sky survey, (2) we

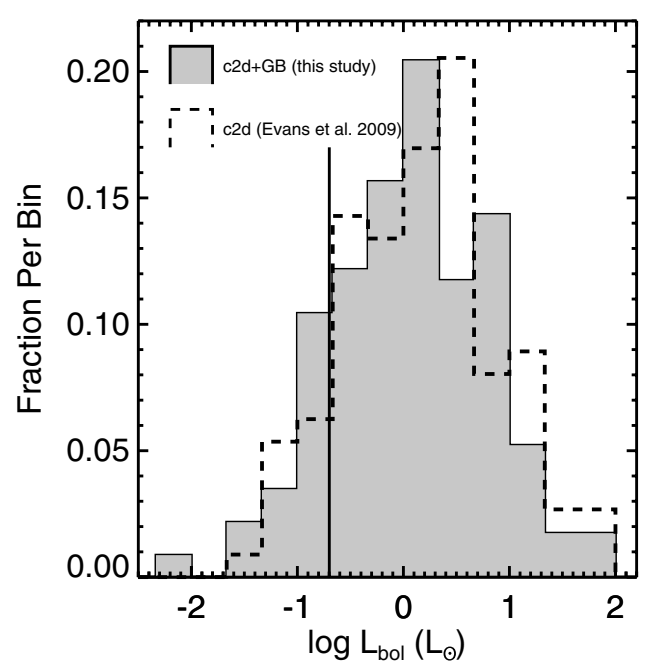

Figure 3. Histogram showing $L_{\mathrm{bol}}$ distributions in $\log$ space with $1 / 3$ dex bins. The shaded histogram shows the distribution derived in this study for the 230 protostars in the combined $\mathrm{c} 2 \mathrm{~d}$ and GB samples (see Figure 1 for error bars). The dashed histogram shows the distribution for the 112 protostars in the $c 2 d$ sample as derived by Evans et al. (2009). The solid vertical line shows the approximate completeness limit of $0.2 L_{\odot}$ for the c $2 d+G B$ sample.

have included additional SHARC-II $350 \mu \mathrm{m}$ photometry from a targeted survey of nearby, low-mass star-forming regions (Wu et al. 2007; M. M. Dunham et al., in preparation) that was not yet available when Evans et al. (2009) completed their study, and (3) we have not included any IRAS photometry. The last change is motivated by the superiority of WISE 12 and $22 \mu \mathrm{m}$ and Spitzer $70 \mu \mathrm{m}$ data to IRAS 12, 25, and $60 \mu \mathrm{m}$ in essentially all cases, and the extreme confusion from both nearby sources and ambient cloud emission in the IRAS $100 \mu \mathrm{m}$ data.

Figure 3 compares the $L_{\text {bol }}$ distributions from this work and from Evans et al. (2009). The new distribution obtained in this study has a similar shape and extent to the c2d-only distribution, except now with better statistics. The medians are also quite similar, with values of $1.3 L_{\odot}$ in this work and $1.5 L_{\odot}$ in the c2d-only sample (Evans et al. 2009). A K-S test on the two distributions returns a value of 0.33 , indicating they are not significantly different.

Despite their general similarities, the two distributions do have different means: $4.3 L_{\odot}$ in this study versus $5.3 L_{\odot}$ in the c2d-only sample (Evans et al. 2009). The mean is strongly influenced by the highest luminosity sources, several of which were overestimated by Evans et al. (2009). To understand the cause of this overestimate, we note that there are 14 sources in the Evans et al. sample saturated at $24 \mu \mathrm{m}$ with Spitzer and thus lacking any photometry between 8 and $70 \mu \mathrm{m}$. By including WISE 12 and $22 \mu \mathrm{m}$ photometry, which was not available to Evans et al., our updated sample fills in this gap. Figure 4 plots the SED for NGC 1333-IRAS2A (source 144 in Table 2), one of the 14 sources saturated in the Spitzer $24 \mu \mathrm{m}$ observations. The SED is plotted as $S_{v}$ versus $v$ in linear space rather than the more typical $v S_{v}$ versus $\lambda$ in log space since the former is the space in which the integral in Equation (2) is calculated. The light shaded area shows the result of the integral when the WISE 12 and $22 \mu \mathrm{m}$ photometry is included, whereas the dark shaded area shows the extra amount added to the integral when no photometry is available between 8 and $70 \mu \mathrm{m}$.

As clearly demonstrated by Figure 4, omitting photometry between 8 and $70 \mu \mathrm{m}$ can lead to significant overestimates of $L_{\text {bol }}$. In the specific case of NGC 1333-IRAS2A, Evans et al. (2009) measured $L_{\text {bol }}=76 L_{\odot}$ whereas we measure 


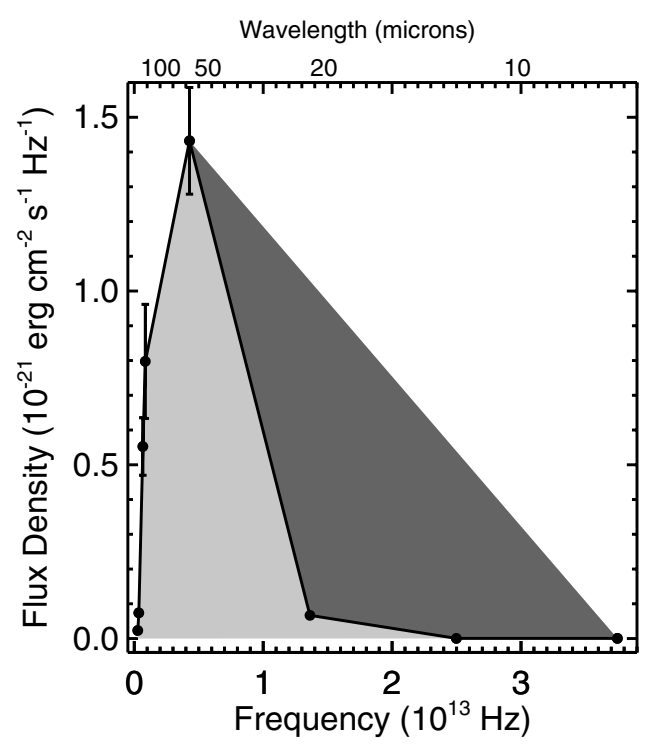

Figure 4. Spectral energy distribution of NGC 1333-IRAS2A, plotted as $S_{v}$ vs. $v$ in linear space. The light shaded area shows the result of integrating under the curve when the WISE 12 and $22 \mu \mathrm{m}$ photometry is included, whereas the dark shaded area shows the extra amount added to the integral when no photometry is available between 8 and $70 \mu \mathrm{m}$, as was the case for Evans et al. (2009).

$L_{\text {bol }}=22 L_{\odot}$ with the WISE 12 and $22 \mu \mathrm{m}$ photometry included, a factor of 3.4 lower. Our measurement is consistent with previous measurements of $L_{\mathrm{bol}}$ for this source whereas the Evans et al. value is not (e.g., Jørgensen et al. 2002). For the 14 sources saturated at $24 \mu \mathrm{m}$, Evans et al. (2009) measured $L_{\text {bol }}$ ranging from 2.6 to $76 L_{\odot}$, with a mean and median of 30 and $27 L_{\odot}$, respectively. For those same 14 sources and with WISE 12 and $22 \mu \mathrm{m}$ photometry included, we measure $L_{\mathrm{bol}}$ ranging from 1.4 to $63 L_{\odot}$, with a mean and median of 20 and $13 L_{\odot}$, respectively. Most of the decrease in the overall sample mean from $5.3 L_{\odot}$ to $4.3 L_{\odot}$ is a result of correcting this overestimate for several relatively high luminosity sources.

\subsubsection{Comparison to Kryukova Results}

Recently, Kryukova et al. (2012) presented observed protostellar luminosity distributions assembled from Spitzer observations of 11 molecular clouds: the $7 \mathrm{c} 2 \mathrm{~d}$ clouds (Chamaeleon II, Lupus I, Lupus III, Lupus IV, Ophiuchus, Perseus, and Serpens), Taurus, and 3 massive star-forming clouds (Orion, Cep OB3, and Mon R2). In total they identified 727 protostars in these clouds. Figure 5 compares our results.

The left panel of Figure 5 compares the $L_{\mathrm{bol}}$ distributions from this work and from Kryukova et al. (2012). We use the contamination-subtracted $L_{\mathrm{bol}}$ distributions from Kryukova et al. for this comparison. The two distributions are generally quite similar, but since Kryukova et al. include three massive starforming clouds in their sample, environmental effects may mask our ability to properly compare the two results. Thus, the right panel of Figure 5 compares the $L_{\mathrm{bol}}$ distributions from this work and from Kryukova et al., where now both samples are restricted to the clouds common to both samples (the c2d clouds).

Inspection of the right panel of Figure 5 clearly shows that, for the same clouds, Kryukova et al. (2012) find an observed distribution of protostellar luminosities that is generally shifted to lower luminosities compared to our results, with a much lower mean $\left(2.3 L_{\odot}\right.$ versus $4.2 L_{\odot}$ in our sample) and much higher fraction of protostars with $L_{\mathrm{bol}} \leqslant 0.1 L_{\odot}$ (22\% versus $7 \%$ in our sample). A K-S test on the two distributions returns a value of 0.01 , verifying that the two distributions are statistically different. Kryukova et al. do not compile complete SEDs to use in calculating bolometric luminosities. Instead, for all sources they identify as protostars, they calculate $L_{\mathrm{MIR}}$, the mid-infrared luminosity from their 2MASS and Spitzer 1.25-24 $\mu \mathrm{m}$ data, and $\alpha$, the infrared spectral index calculated from 3.6 to $24 \mu \mathrm{m}$. For sources with $\alpha \geqslant 0.3$ common to both their sample of protostars and the Evans et al. (2009) sample, they then derive the following empirical relationship:

$$
\frac{L_{\mathrm{MIR}}}{L_{\mathrm{bol}}}=(-0.466 \pm 0.014 \times \log (\alpha)+0.337 \pm 0.053)^{2}
$$
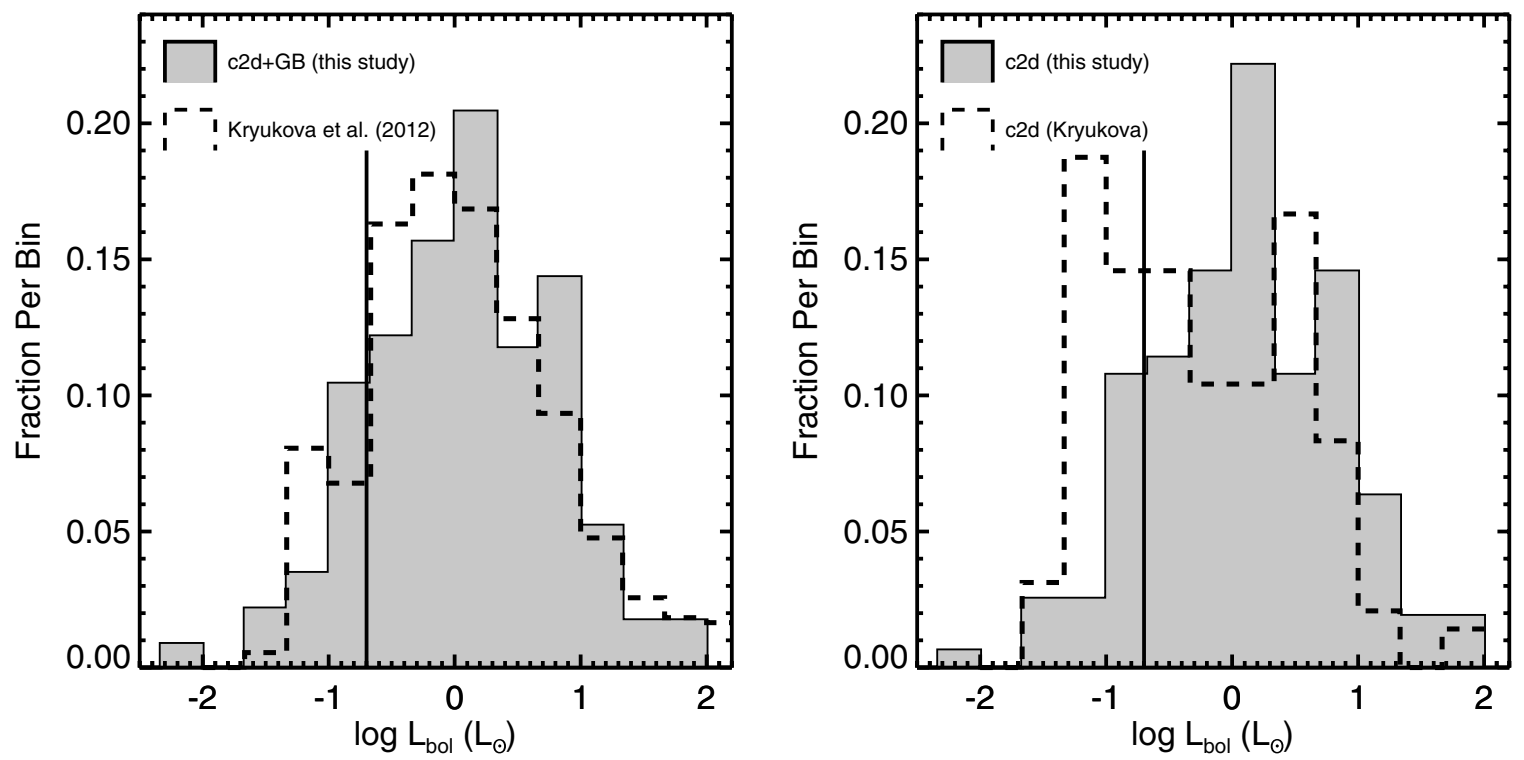

Figure 5. Histogram showing $L_{\text {bol }}$ distributions in $\log$ space with $1 / 3$ dex bins. In both panels, the solid vertical lines show the approximate completeness limits of $0.2 L_{\odot}$ for the c2d+GB sample. Left: the shaded histogram shows the distribution derived in this study for the 230 protostars in the combined c2d and GB samples (see Figure 1 for error bars). The dashed histogram shows the contamination-subtracted distribution for the 727 protostars identified by Kryukova et al. (2012). Right: the shaded histogram shows the distribution from this work when only including the sources in the $\mathrm{c} 2 \mathrm{~d}$ clouds. The dashed histogram shows the contamination-subtracted distribution from Kryukova et al. (2012) when only including sources from the same c2d clouds. 

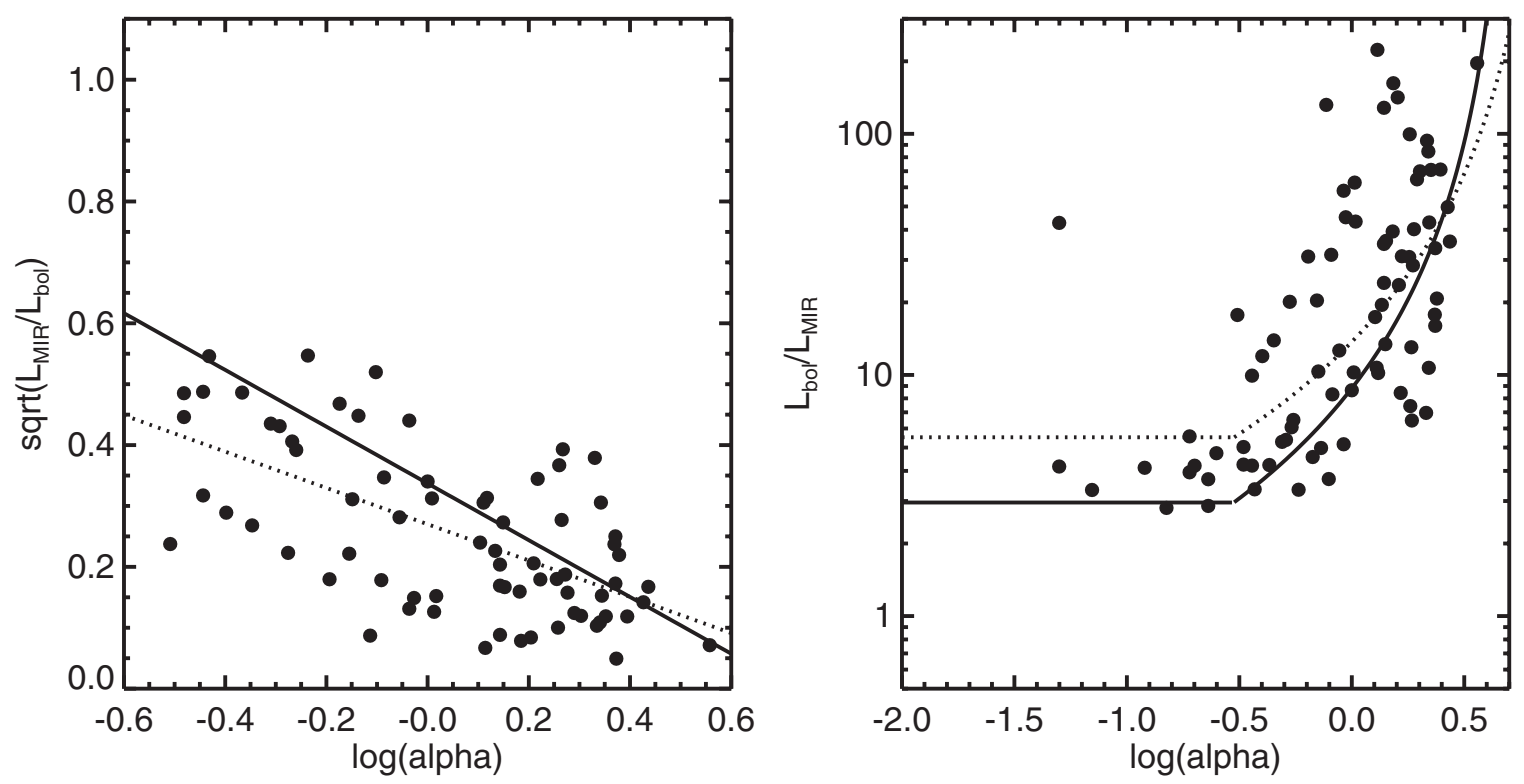

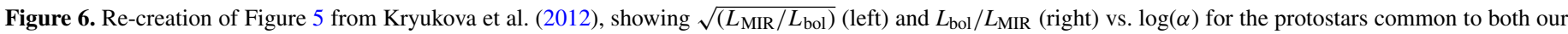

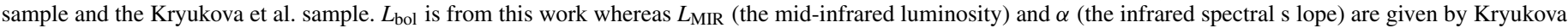

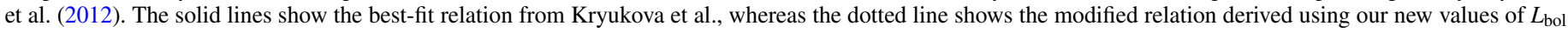
(see text for details).

where $L_{\mathrm{bol}}$ is from Evans et al. (2009). They use this relation to calculate $L_{\mathrm{bol}}$ for all protostars with $\alpha \geqslant 0.3$, and the value of this relation at $\alpha=0.3$ to calculate $L_{\mathrm{bol}}$ for all protostars with $\alpha<0.3$. At least some of the discrepancy between our results and those of Kryukova et al. may arise because we have made several changes to the SEDs used to calculate $L_{\mathrm{bol}}$, as described above in Section 4.1.1. To examine this possibility, we re-derived the above empirical correlation using our new values of $L_{\mathrm{bol}}$ for the sources common to both our sample and the Kryukova et al. sample, and obtained the following modification using a linear least-squares fit:

$$
\frac{L_{\mathrm{MIR}}}{L_{\mathrm{bol}}}=(-0.298 \pm 0.046 \times \log (\alpha)+0.270 \pm 0.013)^{2} .
$$

We illustrate the effects of this modification in Figure 6, which re-creates Figure 5 from Kryukova et al. (2012).

Using our modified relationship between $L_{\mathrm{bol}}, L_{\mathrm{MIR}}$, and $\alpha$, the changes to the Kryukova et al. (2012) $L_{\mathrm{bol}}$ values range from an increase by a factor of 1.9 to a decrease by a factor of 2 , depending on $\alpha$ for each source. The mean change of all sources common to both samples is an increase by a factor of 1.5 . Such a change can explain much of the difference in means in the two samples $\left(4.2 L_{\odot}\right.$ in this study compared to $2.3 L_{\odot}$ in Kryukova et al.), but cannot fully explain the excess of low-luminosity sources. Instead, the remainder of the discrepancy between our results lies in source selection.

Kryukova et al. identify 43 protostars in the c2d clouds not identified by us or by Evans et al. (2009). Figure 7 shows the $L_{\mathrm{bol}}$ distribution for these 43 sources. Most have $L_{\mathrm{bol}} \leqslant 1.0 L_{\odot}$, and while the effects of these "extra" low-luminosity sources are significantly mitigated by statistical contamination corrections included by Kryukova et al., their net effect is to cause an excess of low-luminosity sources compared to our results. The main difference between our method of selecting protostars and that of Kryukova et al. is our requirement of at least one detection at $\lambda \geqslant 350 \mu \mathrm{m}$ to ensure association with dense cores. Since the 43 sources shown in Figure 7 are not in our sample,

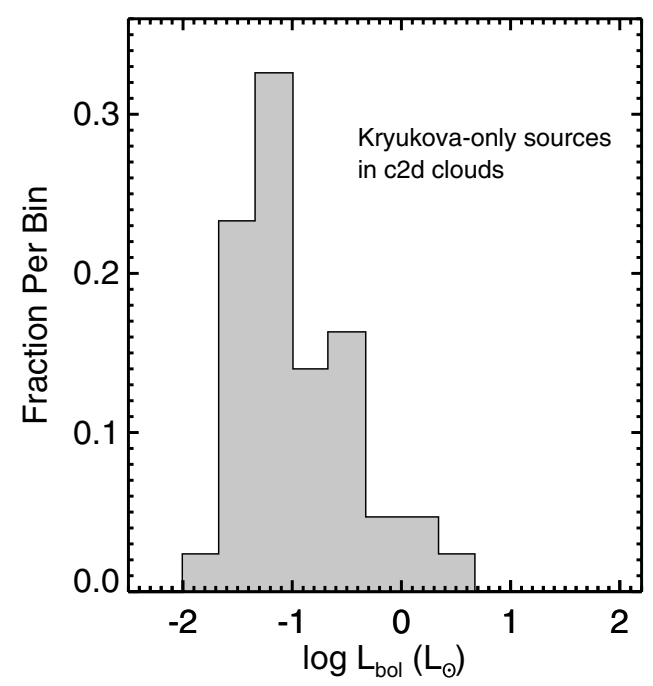

Figure 7. Histogram showing the $L_{\text {bol }}$ distribution in log space for the 43 protostars in the c2d clouds identified by Kryukova et al. (2012) but not by us or by Evans et al. (2009). The bins are 1/3 dex wide.

they are not associated with (sub)millimeter detections and thus not associated with known dense cores. By our definition of a protostar (see Section 1), these are not protostars.

However, it is possible that at least some of these sources are in fact protostars associated with relatively low-mass cores not detected by the (sub)millimeter surveys we used to compile complete SEDs. This is supported by the fact that many such surveys have relatively high completeness limits (for example, the $50 \%$ completeness limits for the Bolocam $1.1 \mathrm{~mm}$ surveys of Perseus, Serpens, and Ophiuchus are 0.8, 0.6, and 0.5 $M_{\odot}$, respectively; Enoch et al. (2008)). On the other hand, some sources may be contaminants masquerading in the sample. Kryukova et al. (2012) made a careful attempt to correct for such contamination in a statistical sense, but the resulting corrections are highly uncertain and may have been underestimated. For example, they applied their protostar selection criteria to the 
catalog produced by the Spitzer SWIRE Legacy survey of the ELAIS N1 extragalactic field (Lonsdale et al. 2003) to estimate the contamination from galaxies and remove the effects of this contamination from their luminosity distribution. Such an estimate is a lower limit only because it does not take into account the fact that galaxies in their cloud source catalogs are observed through the extra extinction of the cloud itself, reddening all galaxies and thus increasing the number of galaxies with their assumed colors of protostars. Furthermore, Heiderman et al. (2010) recently showed that many sources selected as Class I YSOs by their infrared colors lacked the presence of warm, dense gas and are thus not protostars (and may not even be YSOs at all; see Heiderman et al. for details). Since Kryukova et al. used similar color-based selection criteria, it is plausible that not all of their sources are actually protostars. Our (sub)millimeter detection requirement should remove such fake sources.

Ultimately, we conclude that there are limitations to both our method and that used by Kryukova et al. We are limited by the availability and sensitivity of (sub)millimeter surveys whereas they are limited by uncertain statistical corrections for contamination. We prefer our method because it ensures a reliable sample, but the Kryukova et al. (2012) results emphasize that this reliability may come at the expense of completeness. Some of the "extra" 43 sources in the c 2 d clouds they identify but we do not may in fact be real protostars. Quantifying how many is simply not possible until future surveys in the far-infrared and submillimeter become available, as discussed further in Section 5.1.

\subsection{Theoretical Investigations of Protostellar Luminosities}

With the new observed protostellar luminosity distributions derived from large Spitzer surveys (Evans et al. 2009; Kryukova et al. 2012; this work), several recent studies comparing the predictions of theoretical accretion processes to the observations have been published. In one such study, Dunham \& Vorobyov (2012) coupled two-dimensional radiative transfer calculations with the numerical hydrodynamical simulations of Vorobyov \& Basu (2005, 2006, 2010). These simulations predict accretion rates that both generally decline with time and feature shortterm variability and episodic bursts caused by disk gravitational instability and fragmentation. Dunham \& Vorobyov (2012) used the core, disk, and protostellar masses, radii, and mass accretion rates predicted by the simulations as inputs to their radiative transfer calculations. They included the effects of external heating in their radiative transfer models, and calculated model SEDs at all inclinations from the beginning of collapse until the end of the embedded phase. They used these SEDs to calculate $L_{\mathrm{bol}}$ in the same manner as observers (Equation (2)) at all inclinations and all time steps. Finally, they assembled a theoretical prediction of the observed luminosity distribution by calculating the fraction of total time the models spend at each $L_{\text {bol }}$, weighted by inclination and initial core mass.

Dunham \& Vorobyov compared their model luminosity distribution to the c2d observations presented by Evans et al. (2009) and showed that the models generally match the shape and spread of the observed luminosity distribution, indicating that the underlying variable accretion process predicted by the Vorobyov \& Basu $(2005,2006,2010)$ simulations offers a possible resolution of the luminosity problem. Figure 8 compares our new observations with the Dunham \& Vorobyov (2012) models. As our results are generally similar to Evans et al. (2009) in terms of the shape and extent of the observed luminosity

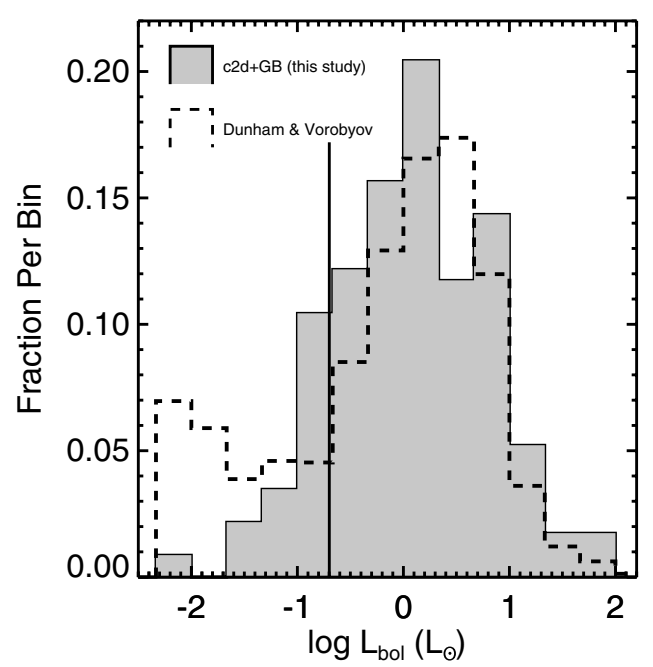

Figure 8. Histogram showing $L_{\mathrm{bol}}$ distributions in $\log$ space with $1 / 3$ dex bins. The shaded histogram shows the distribution derived in this study for the 230 protostars in the combined c $2 \mathrm{~d}$ and GB samples (see Figure 1 for error bars). The dashed histogram shows the model distribution derived by Dunham \& Vorobyov (2012) based on a set of hydrodynamical simulations predicting accretion rates that both generally decline with time and feature short-term variability and episodic bursts caused by disk gravitational instability and fragmentation. The solid vertical line shows the approximate completeness limit of $0.2 L_{\odot}$ for the c2d+GB sample.

distribution (see Section 4.1.1), except with improved statistics, the basic conclusions of Dunham \& Vorobyov are unchanged. While the agreement is not perfect, the models provide a reasonable match to the observed luminosity distribution, with a $\mathrm{K}-\mathrm{S}$ test on the two distributions returning a value of 0.41 . The only significant discrepancy between our observations and the Dunham \& Vorobyov (2012) models is at $L_{\text {bol }} \lesssim 0.2 L_{\odot}$. As discussed in detail by Dunham \& Vorobyov (2012), this $L_{\text {bol }}$ regime is at or below the completeness limit of the $\mathrm{c} 2 \mathrm{~d}$ and GB surveys, rendering a proper comparison of observed and model $L_{\text {bol }}$ impossible at such luminosities (see also Section 5.3). The variable accretion process predicted by the Vorobyov \& Basu $(2005,2006,2010)$ simulations and considered by Dunham \& Vorobyov (2012) remains a valid solution to the luminosity problem.

Offner \& McKee (2011) have also presented a recent theoretical study of protostellar luminosities. They derived analytic luminosity functions for several different accretion scenarios and compared their results to the c2d observations presented by Evans et al. (2009). In addition to comparing to the mean and median of the observed distribution, they calculated four dimensionless quantities to characterize their luminosity functions and compared to the same quantities calculated from the observations. Their study was our motivation for calculating and tabulating the quantities listed in the last four rows of Table 3, although we emphasize that the last quantity presented in Table 3 , the fraction of protostars with $L_{\mathrm{bol}} \leqslant 0.1 L_{\odot}$, is not the same as the fraction of VeLLOs from Offner \& McKee (2011). Their quantity was calculated as the ratio of sources with $L_{\text {bol }} \leqslant 0.14 L_{\odot}$ to those with $L_{\text {bol }} \leqslant 1.4 L_{\odot}$ and was chosen to provide a direct comparison to the observations presented by Dunham et al. (2008).

Based on comparing their analytic luminosity functions to the c2d observations, Offner \& McKee (2011) concluded that accretion scenarios that tend toward a constant accretion time rather than a constant accretion rate are better able to match the 
observed protostellar luminosity distribution. Given the similarity of our observed luminosity distribution to that of the $\mathrm{c} 2 \mathrm{~d}$ sample, the new observations presented here do little to change the findings of Offner \& McKee. Their conclusion is in general agreement with a series of investigations by Myers (2010, 2011, 2012), who derived analytic luminosity distributions based on simple models of protostellar evolution assuming constant protostellar birth rates, accretion from both the dense core and from the surrounding ambient medium ("core-clump" accretion, to use their terminology), and accretion durations set by the assumption of an equally likely stopping time. They showed that such models, which predict accretion rates that increase with protostellar mass and thus tend toward a constant accretion time rather than constant accretion rate, exhibit good agreement with observed protostellar luminosities.

While the Dunham \& Vorobyov (2012), Offner \& McKee (2011), and Myers (2010, 2011, 2012) models have all succeeded in matching the observed distribution of protostellar luminosities, they do so with models featuring very different accretion properties. Like the collapse of a singular isothermal sphere, which does not match observations, the Dunham \& Vorobyov (2012) models feature time-averaged accretion rates (averaged over the full duration of the embedded phase) that do not vary with the final mass of the protostar. In other words, higher mass protostars require more time to form than lowmass protostars. Their solution to the luminosity problem is to invoke variability and episodic accretion bursts as predicted by the Vorobyov \& Basu $(2005,2006,2010)$ simulations, which reduce the accretion rates for most times and increase them during short-lived accretion bursts. On the other hand, Offner \& McKee (2011) and Myers (2010, 2011, 2012) solve the luminosity problem by adopting models with time-averaged accretion rates that increase with the final mass of a protostar, so that all protostars form in about the same amount of time regardless of their final mass. These studies emphasize that there is more than one possible resolution to the luminosity problem, and future theoretical work is needed to better decipher the implications of protostellar luminosities for the underlying mass accretion process and to distinguish between these different accretion scenarios. In this work we have assembled a larger data set to which such work should compare.

One weakness of both the Offner \& McKee (2011) and Myers $(2010,2011,2012)$ models is that they do not predict observed protostellar luminosities. The observed luminosity of a protostar includes accretion luminosity, photosphere luminosity, and external luminosity from heating by the interstellar radiation field, and is dependent on the physical structure and inclination of the source. Offner \& McKee (2011) only include the two internal luminosity components (accretion and photosphere), and Myers (2010, 2011, 2012) include only accretion luminosity. Furthermore, neither set of models takes into account the effects of source structure and inclination. The evolutionary radiative transfer models presented by Dunham \& Vorobyov (2012) do take all these effects into account and calculate observed luminosities before comparing to observations, but they can also be criticized for assuming a very simple physical structure, not allowing for any variation in this structure, adopting a fixed interstellar radiation field with no variation in its strength, attenuation, or spectral shape, and weighting by only one of several formulations of the stellar initial mass function. Future theoretical work must build on the foundations laid by these recent studies to properly compare theoretical predictions to observed protostellar luminosities.

\subsection{Effects of Including FIR/SMM Photometry}

The SEDs of embedded sources typically peak around 100-300 $\mu \mathrm{m}$ (e.g., André et al. 1993; Enoch et al. 2009; Dunham et al. 2008); accurate sampling in the far-infrared and submillimeter is thus necessary to ensure accurate measurements of $L_{\text {bol }}$. Missing photometry near the peaks of the SEDs will result in underestimates of $L_{\text {bol }}$. As described above in Section 2.3, we included Spitzer $160 \mu \mathrm{m}$ photometry for sources detected and not located in saturated or confused regions, SHARC-II $350 \mu \mathrm{m}$ photometry when available from a targeted survey of protostellar sources (Wu et al. 2007; M. M. Dunham et al., in preparation), and SCUBA $450 \mu \mathrm{m}$ photometry when available in the SCUBA Legacy Catalog (Di Francesco et al. 2008). Out of the 230 total protostars identified in this work, $130(57 \%)$ include at least one photometry point at 160,350 , or $450 \mu \mathrm{m}$. The other $100(43 \%)$ lack any available photometry between $70 \mu \mathrm{m}$ and at least $850 \mu \mathrm{m}$, and sometimes between $70 \mu \mathrm{m}$ and $1.1 \mathrm{~mm}$. The last column of Table 2 indicates whether each protostar has any such photometry available.

How much have we underestimated $L_{\mathrm{bol}}$ for the $43 \%$ of the sample lacking any far-infrared and submillimeter photometry, and what effect does this have on the derived luminosity distribution? To address these questions, the left panel of Figure 9 plots the $L_{\text {bol }}$ distribution separately for the sources with and without at least one observed photometry point at 160,350 , or $450 \mu \mathrm{m}$. As expected, the $L_{\text {bol }}$ distribution for sources without any far-infrared or submillimeter data is shifted to lower luminosities. To quantify the amount by which $L_{\text {bol }}$ is underestimated for these sources, we took the 130 protostars with at least one such observed photometry point, removed all data between 70 and $850 \mu \mathrm{m}$, and recalculated $L_{\mathrm{bol}}$. The results are shown in the right panel of Figure 9, which plots the ratio of $L_{\text {bol }}$ calculated with all available photometry to $L_{\text {bol }}$ calculated with all data between 70 and $850 \mu \mathrm{m}$ removed versus $T_{\text {bol }}$. The mean and median of this ratio are 2.6 and 1.5, with a few sources showing underestimates in $L_{\mathrm{bol}}$ up to factors of 8-10. There may be some evidence for a trend of larger $L_{\mathrm{bol}}$ underestimates for more deeply embedded sources (those with lower $T_{\text {bol }}$ ). Such a trend is not surprising since a greater fraction of the total luminosity is emitted in the far-infrared and submillimeter for more deeply embedded sources, but, if present, the trend is not very strong. Even sources with a $T_{\text {bol }}$ of several hundred kelvin can have $L_{\text {bol }}$ underestimated by a factor of two or more.

Another way of examining the effects of missing far-infrared and submillimeter data is to use Herschel 100-500 $\mu$ m photometry. The Herschel Gould Belt survey is currently surveying all of the clouds included here and will eventually provide such photometry (André et al. 2010). Maury et al. (2011) recently used data from this survey to study the protostellar population of part of Aquila. The ideal comparison would be to include Herschel photometry in our SEDs and recalculate $L_{\text {bol }}$ for each source, but we are unable to do this since Maury et al. did not publish the Herschel fluxes. They did, however, publish their own measurement of $L_{\mathrm{bol}}$ for each source including the Herschel data. We have identified 17 protostars in Aquila that overlap with their sample; in all 17 cases we lacked any farinfrared or submillimeter photometry. Figure 10 plots the ratio of $L_{\text {bol }}$ from Maury et al. (2011) with the Herschel data included to $L_{\text {bol }}$ from this work without the Herschel data versus $T_{\text {bol }}$ from Maury et al. (2011) with the Herschel data included. The results are strikingly similar to our above analysis: including the Herschel far-infrared and submillimeter photometry increases 

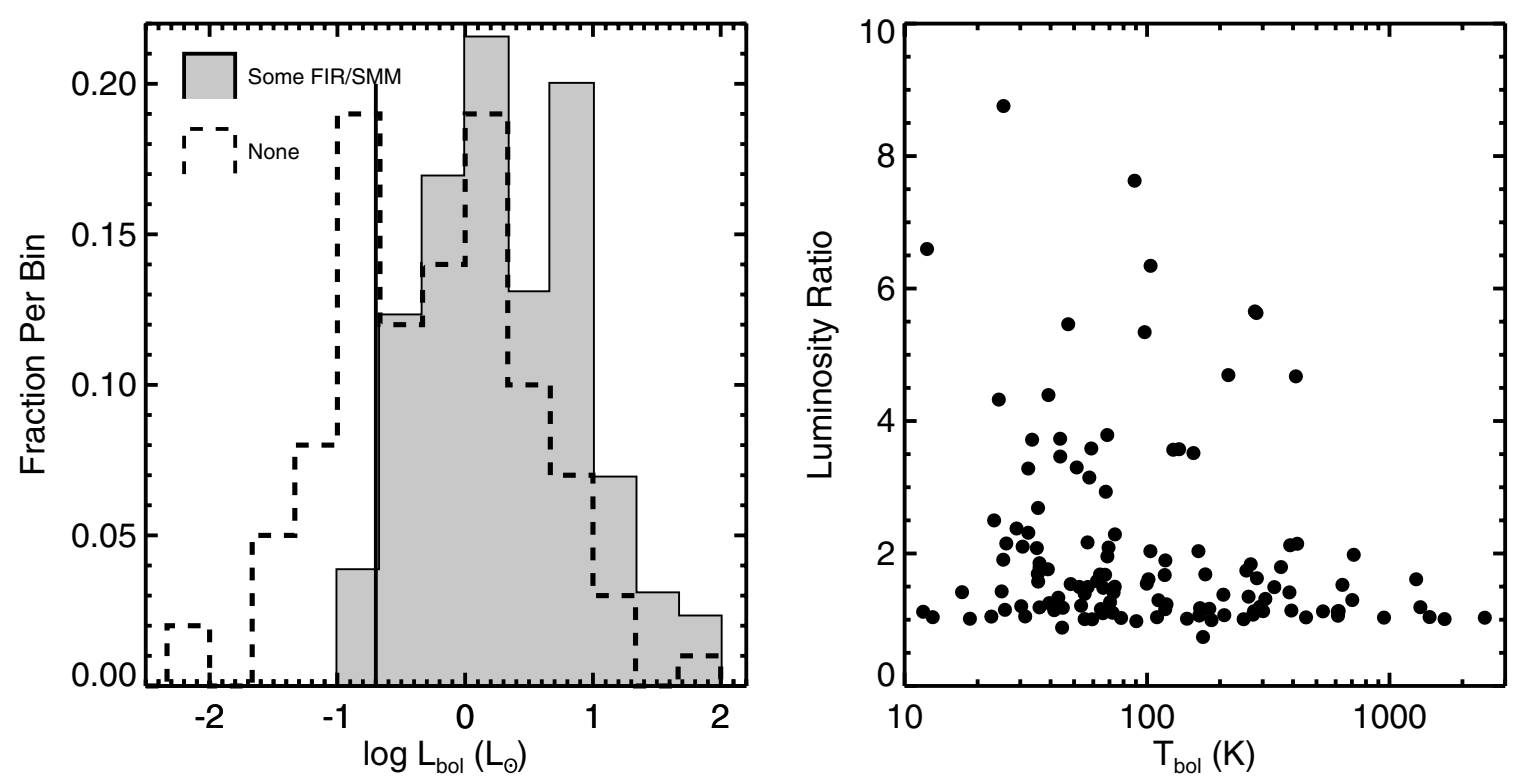

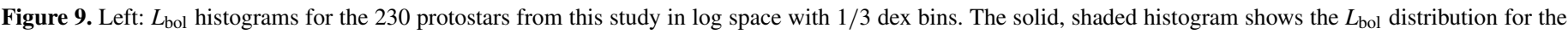

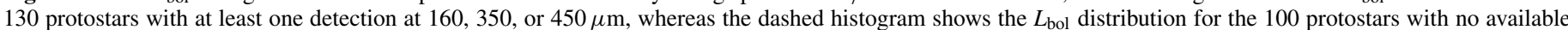

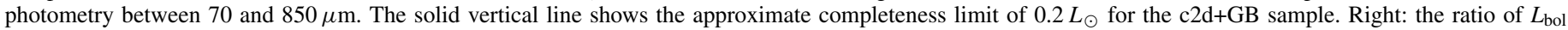

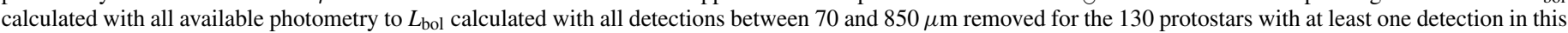
wavelength range, plotted vs. $T_{\text {bol }}$.

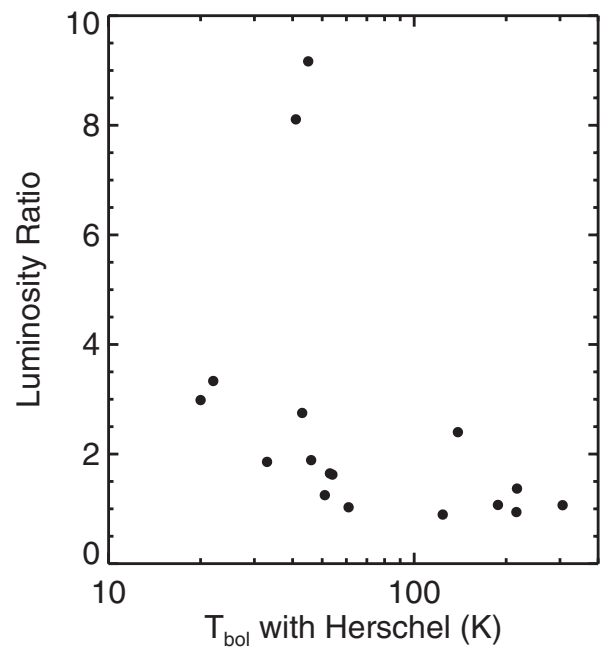

Figure 10. The ratio of $L_{\mathrm{bol}}$ with Herschel photometry to $L_{\mathrm{bol}}$ without Herschel photometry vs. $T_{\text {bol }}$ calculated with Herschel photometry for the 17 protostars in Aquila. $L_{\text {bol }}$ and $T_{\text {bol }}$ with Herschel photometry are taken from Maury et al. (2011) and $L_{\mathrm{bol}}$ without Herschel photometry is calculated in this work with the observed photometry (see text for details).

$L_{\text {bol }}$ by a mean and median of 2.6 and 1.6, respectively, and by up to factors of 8-10 in some cases. Furthermore, there is again some evidence of a trend with $T_{\text {bol }}$, but the sample size is too small for robust statistics. This analysis should be repeated for a larger sample and with $L_{\text {bol }}$ calculated in a consistent manner with and without the Herschel photometry, but the agreement with the above analysis is encouraging.

Based on two separate investigations, we conclude that lacking any far-infrared or submillimeter photometry will lead to underestimates in $L_{\text {bol }}$ by a factor of about 2.5 on average, but up to factors of 8-10 in extreme cases. These findings are consistent with earlier results by Dunham et al. (2008) and Enoch et al. (2009), who also investigated this topic. Since nearly half $(43 \%)$ of our sample currently lacks any such photometry, including Herschel photometry when it becomes available will affect our derived luminosity distribution. To determine how significant this effect will be, we increased the calculated $L_{\text {bol }}$ for all 100 sources lacking any far-infrared or submillimeter photometry by factors of 2.5 and found that the mean and median of the full sample of 230 sources increase to 5.8 and $1.8 L_{\odot}$, respectively. Compared to the values listed in Table 3, both quantities increase by $35 \%-40 \%$. Testing this conclusion and determining the full effects on the shape and extent of the luminosity distribution can only be done once the Herschel Gould Belt survey is complete and the photometry is available.

\section{FUTURE WORK}

\subsection{Completeness and Reliability of the Sample}

In order to be classified as a protostar, we require at least one detection at $\lambda \geqslant 350 \mu \mathrm{m}$ to signify the presence of a dense core. However, as described in Section 2.3, we only have access to complete (sub)millimeter surveys of 6 out of the 18 clouds (Chamaeleon I, Chamaeleon II, Chamaeleon III, Ophiuchus, Perseus, and Serpens), plus a partial survey of Aquila (Maury et al. 2011) and piecemeal coverage of other clouds from the SCUBA Legacy Catalog (Di Francesco et al. 2008). We are almost certainly missing protostars from the clouds where we have incomplete (sub)millimeter coverage. Furthermore, where we do have coverage, the surveys are typically incomplete to low-mass cores with $M \lesssim 0.5 M_{\odot}$ (e.g., Enoch et al. 2008). We are also likely missing some protostars in low-mass cores.

Kryukova et al. (2012) have also studied the protostellar luminosity distribution. They do not require a (sub)millimeter detection to be included in their sample. In a detailed comparison between our results and their results (see Section 4.1.2) we showed that they identify approximately $40 \%$ more protostars than we do in the same clouds and that these "extra" protostars mostly have $L_{\text {bol }} \leqslant 1.0 L_{\odot}$. Some may be true protostars located in low-mass cores below the sensitivities of the (sub)millimeter surveys available to us, but some may also be contaminants masquerading as protostars in their sample. 
Based on the presently available data, we are unable to evaluate how significantly our sample lacks completeness and how significantly the Kryukova et al. (2012) sample lacks reliability. Both should be fully resolved once the James Clerk Maxwell Telescope SCUBA-2 Legacy (Ward-Thompson et al. 2007) and Herschel (André et al. 2010) Gould Belt Surveys are complete, since the two surveys together will fully characterize the population of dense cores in all of the Gould Belt clouds with sensitivities below $0.1 M_{\odot}$. Future work must revisit the topic of defining the sample of protostars once the results from these surveys are available.

\subsection{Accuracy of $L_{b o l}$ Calculation}

As discussed in detail in Section 4.3, nearly half (43\%) of our sample lacks any detections between $70 \mu \mathrm{m}$ and at least $850 \mu \mathrm{m}$, and sometimes between $70 \mu \mathrm{m}$ and $1.1 \mathrm{~mm}$. We have shown that such sources will have their $L_{\text {bol }}$ underestimated by a factor of about 2.5 , on average, and up to factors of 8-10 in extreme cases. We have estimated that correcting these underestimates will increase both the mean and median protostellar luminosity by about $35 \%-40 \%$. Verifying this estimate and examining the full effects of these underestimates on the shape and extent of the luminosity distribution must be revisited once the Herschel Gould Belt survey is complete and provides $100-500 \mu \mathrm{m}$ photometry for our full sample.

\subsection{Extending to Lower Luminosities}

As discussed in Section 3, our protostellar sample is generally only complete for $L_{\mathrm{bol}}>0.2 L_{\odot}$, with the exact completeness limit different for each source depending on its distance, detailed spectral shape, and amount of external heating. Does there exist a population of protostars with luminosities below the completeness of the $\mathrm{c} 2 \mathrm{~d}$ and Gould Belt surveys?

Recent evidence suggests that the answer is yes. First, in a detailed study of the population of low luminosity protostars in the c2d clouds, Dunham et al. (2008) noted that protostars were found all the way down to the sensitivity limit and suggested that the lower limit to protostellar luminosities had not yet been found. Second, very recent work has identified a population of cores originally believed to be starless and undetected in c2d and other Spitzer observations of similar depth but found to be driving molecular outflows through sensitive (sub)millimeter interferometer observations. To date, seven such objects have been identified, and all but one are located in the Perseus Molecular Cloud (Chen et al. 2010, 2012; Enoch et al. 2010; Dunham et al. 2011; Pineda et al. 2011; Schnee et al. 2012; Pezzuto et al. 2012).

Both the true number and evolutionary status of these objects remains unknown. Most have been discovered serendipitously through (sub)millimeter interferometer detections of outflows in observations targeting cores believed to be starless. A full survey for such objects has not been possible to date due to the prohibitively large time requests that would be required, although such surveys should be possible in the very near future with ALMA. None of the sources were detected in the infrared with c2d or other Spitzer surveys of similar depth, implying upper limits of $0.01-0.1 L_{\odot}$ for both $L_{\text {int }}$ and $L_{\text {bol }}$. One source was detected in very deep, targeted Spitzer $70 \mu \mathrm{m}$ observations and found to have $L_{\text {int }} \sim 0.01 L_{\odot}$ and $L_{\text {bol }} \sim 0.2 L_{\odot}$ (Enoch et al. 2010). They have all been proposed as candidate first hydrostatic cores, a short-lived stage between the starless and protostellar phases (Larson 1969). First cores can drive outflows, although there is still debate about the physical properties of such outflows and which, if any, of the sources have outflow properties consistent with theoretical predictions (e.g., Machida et al. 2008; Dunham et al. 2011; Price et al. 2012). Future work must be devoted to determining how many of these objects exist, their true evolutionary status, and the effects their existence will have on the protostellar luminosity distribution.

\section{SUMMARY}

In this paper we have studied the protostellar luminosity distribution based on data from two Spitzer Legacy surveys of nearby star-forming regions. We summarize our main results as follows.

1. Starting from a list of 2966 YSOs identified via their positions in various color-color and color-magnitude diagrams, we identify 230 protostars in the Spitzer c $2 \mathrm{~d}$ and Gould Belt Legacy surveys based on association with a dense core detected at (sub)millimeter wavelengths. We compile as complete SEDs as possible for all 230 sources, and use these SEDs to calculate $L_{\mathrm{bol}}$ and $T_{\mathrm{bol}}$.

2. The protostellar luminosity distribution extends over three orders of magnitude, from $0.01 L_{\odot}$ to $69 L_{\odot}$, and has a mean and median of $4.3 L_{\odot}$ and $1.3 L_{\odot}$, respectively. Several dimensionless quantities characterizing the shape of the distribution are also calculated and tabulated.

3. The luminosity distributions are generally similar for Class 0 and Class I sources, with mean (median) values of $4.5 L_{\odot}$ and $3.8 L_{\odot}\left(1.4 L_{\odot}\right.$ and $\left.1.0 L_{\odot}\right)$ for the Class 0 and I sources, respectively. The only difference is an excess of low luminosity Class I sources compared to the Class 0 population: $36 \%$ of the Class I sources have $L_{\mathrm{bol}}<0.5 L_{\odot}$ compared to only $20 \%$ for the Class 0 population. A K-S test confirms that this difference is statistically significant.

4. Our derived luminosity distribution is similar to that obtained by Evans et al. (2009) from the c $2 d$ data, except with better statistics. The most significant change is that we have added additional data to improve the accuracy of the $L_{\mathrm{bol}}$ measurement for sources saturated at $24 \mu \mathrm{m}$ with Spitzer, reducing $L_{\text {bol }}$ by factors of $\sim 2-3$ for 14 of the highest luminosity sources. This improvement is responsible for most of the decrease in the mean $L_{\mathrm{bol}}$ from $5.3 L_{\odot}$ in Evans et al. (2009) to $4.3 L_{\odot}$ in this study.

5. Our derived luminosity distribution is significantly different from that of Kryukova et al. (2012), who find a strong excess of sources at $L_{\text {bol }} \leqslant 1.0 L_{\odot}$ compared to our results. Some of this discrepancy can be explained by the fact that we have modified the SEDs used to calculate $L_{\mathrm{bol}}$, requiring a modification to the Kryukova et al. relationship used to calculate $L_{\text {bol }}$, but some is also due to source selection. By not requiring a (sub)millimeter detection they identify nearly $40 \%$ more protostars than we do in the same clouds, most of which are located at low $L_{\text {bol }}$. Future work is needed to better characterize the completeness of our sample and the reliability of the Kryukova et al. sample.

6. Of 230 protostars, $100(43 \%)$ lack any available data in the far-infrared and submillimeter. The calculated $L_{\mathrm{bol}}$ for these sources underestimates the true $L_{\text {bol }}$ by a factor of 2.5 on average, and up to factors of $8-10$ in extreme cases. Including far-infrared and submillimeter data for these sources once they become available from the Herschel Gould Belt survey will likely increase both the mean and median $L_{\text {bol }}$ by $35 \%-40 \%$. 
7. The conclusions of several recent theoretical studies of the protostellar accretion process that compare to the Evans et al. (2009) c2d luminosity distribution remain valid since our results are not substantially different from the $\mathrm{c} 2 \mathrm{~d}$ results. As these studies demonstrate that there is more than one plausible accretion scenario that can match observations, we have emphasized that future theoretical work is needed to better decipher the implications of protostellar luminosities for the underlying mass accretion process.

We believe that the results presented here are the most complete and reliable census of protostellar luminosities assembled to date. Nevertheless, as outlined above in Section 5, several avenues of future work must be pursued to better define the true completeness and reliability of the protostellar sample, more accurately measure $L_{\text {bol }}$ for each source, and better understand the full extent and shape of the low end of the luminosity distribution.

We thank Erin Kryukova, Tom Megeath, Chris McKee, Stella Offner, and Phil Myers for reading a draft in advance of publication and providing helpful comments. This work is based primarily on observations obtained with the Spitzer Space Telescope, operated by the Jet Propulsion Laboratory, California Institute of Technology. It also uses data products from the Two Micron All Sky Survey, which is a joint project of the University of Massachusetts and the Infrared Processing and Analysis Center/California Institute of Technology, funded by the National Aeronautics and Space Administration and the National Science Foundation. These data were provided by the NASA/IPAC Infrared Science Archive, which is operated by the Jet Propulsion Laboratory, California Institute of Technology, under contract with NASA. This research has made use of NASA's Astrophysics Data System (ADS) Abstract Service, and the SIMBAD database, operated at CDS, Strasbourg, France. M.M.D. acknowledges the support of a NASA/JPL Herschel OT1 grant. Additional support was provided by the NSF through grant AST-0845619 to H.G.A. N.J.E. acknowledges support from the NSF through grant AST-1109116.

\section{REFERENCES}

Alcalá, J. M., Spezzi, L., Chapman, N., et al. 2008, ApJ, 676, 427 André, P., Men'shchikov, A., Bontemps, S., et al. 2010, A\&A, 518, L102 André, P., Ward-Thompson, D., \& Barsony, M. 1993, ApJ, 406, 122 Armitage, P. J., Livio, M., \& Pringle, J. E. 2001, MNRAS, 324, 705 Basu, S. 1997, ApJ, 485, 240

Beichman, C. A., Myers, P. C., Emerson, J. P., et al. 1986, ApJ, 307, 337 Belloche, A., Parise, B., Schuller, F., et al. 2011a, A\&A, 535, A2 Belloche, A., Schuller, F., Parise, B., et al. 2011b, A\&A, 527, A145 Bonnell, I., \& Bastien, P. 1992, ApJL, 401, L31

Bourke, T. L., Myers, P. C., Evans, N. J., II, et al. 2006, ApJL, 649, L37 Cha, S.-H., \& Nayakshin, S. 2011, MNRAS, 415, 3319

Chapman, N. L., Lai, S.-P., Mundy, L. G., et al. 2007, ApJ, 667, 288

Chapman, N. L., Mundy, L. G., Lai, S.-P., \& Evans, N. J., II 2009, ApJ, 690, 496

Chen, H., Myers, P. C., Ladd, E. F., \& Wood, D. O. S. 1995, ApJ, 445, 377

Chen, X., Arce, H. G., Dunham, M. M., et al. 2012, ApJ, 751, 89

Chen, X., Arce, H. G., Zhang, Q., et al. 2010, ApJ, 715, 1344

Comerón, F. 2008, in Handbook of Star Forming Regions: Vol. II. The Southern Sky, ed. B. Reipurth (ASP Monographs; San Francisco, CA: ASP), 295

Crapsi, A., van Dishoeck, E. F., Hogerheijde, M. R., Pontoppidan, K. M., \& Dullemond, C. P. 2008, A\&A, 486, 245

Dalba, P. A., \& Stahler, S. W. 2012, MNRAS, 425, 1591

de Geus, E. J., de Zeeuw, P. T., \& Lub, J. 1989, A\&A, 216, 44

Di Francesco, J., Evans, N. J., II, Caselli, P., et al. 2007, in Protostars and Planets V, ed. B. Reipurth, D. Jewitt, \& K. Keil (Tucson, AZ: Univ. Arizona Press), 17
Di Francesco, J., Johnstone, D., Kirk, H., MacKenzie, T., \& Ledwosinska, E. 2008, ApJS, 175, 277

Dowell, C. D., Allen, C. A., Babu, R. S., et al. 2003, Proc. SPIE, 4855, 73

Dunham, M. M., Chen, X., Arce, H. G., et al. 2011, ApJ, 742, 1

Dunham, M. M., Crapsi, A., Evans, N. J., II, et al. 2008, ApJS, 179, 249

Dunham, M. M., Evans, N. J., II, Bourke, T. L., et al. 2006, ApJ, 651, 945

Dunham, M. M., Evans, N. J., Bourke, T. L., et al. 2010a, ApJ, 721, 995

Dunham, M. M., Evans, N. J., II, Terebey, S., Dullemond, C. P., \& Young, C. H. 2010b, ApJ, 710, 470

Dunham, M. M., \& Vorobyov, E. I. 2012, ApJ, 747, 52

Dzib, S., Loinard, L., Mioduszewski, A. J., et al. 2010, ApJ, 718, 610

Dzib, S., Loinard, L., Mioduszewski, A. J., et al. 2011, RMxAC, 40, 231

Enoch, M. L., Evans, N. J., II, Sargent, A. I., \& Glenn, J. 2009, ApJ, 692, 973

Enoch, M. L., Evans, N. J., II, Sargent, A. I., et al. 2008, ApJ, 684, 1240

Enoch, M. L., Glenn, J., Evans, N. J., II, et al. 2007, ApJ, 666, 982

Enoch, M. L., Lee, J.-E., Harvey, P., Dunham, M. M., \& Schnee, S. 2010, ApJL, 722, L33

Enoch, M. L., Young, K. E., Glenn, J., et al. 2006, ApJ, 638, 293

Evans, N. J., II, Allen, L. E., Blake, G. A., et al. 2003, PASP, 115, 965

Evans, N. J., II, Dunham, M. M., Jørgensen, J. K., et al. 2009, ApJS, 181, 321

Evans, N. J., II, Rawlings, J. M. C., Shirley, Y. L., \& Mundy, L. G. 2001, ApJ, 557,193

Evans, N. J., II, Harvey, P. M., Dunham, M. M., et al. 2007, Final Delivery of Data from the c2d Legacy Project: IRAC and MIPS (Pasadena, CA: SSC), http://irsa.ipac.caltech.edu/data/SPITZER/docs/spitzermission/ observingprograms/legacy/

Fatuzzo, M., Adams, F. C., \& Myers, P. C. 2004, ApJ, 615, 813

Fazio, G. G., Hora, J. L., Allen, L. E., et al. 2004, ApJS, 154, 10

Foster, P. N., \& Chevalier, R. A. 1993, ApJ, 416, 303

Galli, D., \& Shu, F. H. 1993a, ApJ, 417, 220

Galli, D., \& Shu, F. H. 1993b, ApJ, 417, 243

Glenn, J., Bock, J. J., Chattopadhyay, G., et al. 1998, Proc. SPIE, 3357, 326

Gutermuth, R. A., Bourke, T. L., Allen, L. E., et al. 2008, ApJL, 673, L151

Gutermuth, R. A., Megeath, S. T., Myers, P. C., et al. 2009, ApJS, 184, 18

Harvey, P. M., Chapman, N., Lai, S.-P., et al. 2006, ApJ, 644, 307

Harvey, P. M., Huard, T. L., Jørgensen, J. K., et al. 2008, ApJ, 680, 495

Harvey, P. M., Merín, B., Huard, T. L., et al. 2007a, ApJ, 663, 1149

Harvey, P. M., Rebull, L. M., Brooke, T., et al. 2007b, ApJ, 663, 1139

Hatchell, J., Terebey, S., Huard, T., et al. 2012, ApJ, 754, 104

Heiderman, A., Evans, N. J., II, Allen, L. E., Huard, T., \& Heyer, M. 2010, ApJ, 723, 1019

Henriksen, R., Andre, P., \& Bontemps, S. 1997, A\&A, 323, 549

Hunter, C. 1977, ApJ, 218, 834

Jørgensen, J. K., Harvey, P. M., Evans, N. J., II, et al. 2006, ApJ, 645, 1246

Jørgensen, J. K., Schöier, F. L., \& van Dishoeck, E. F. 2002, A\&A, 389, 908

Kauffmann, J., Bertoldi, F., Bourke, T. L., et al. 2011, MNRAS, 416, 2341

Kauffmann, J., Bertoldi, F., \& Evans, N. J., II C2D Collaboration. 2005, AN, 326,878

Kenyon, S. J., Gomez, M., Marzke, R. O., \& Hartmann, L. 1994, AJ, 108, 251

Kenyon, S. J., \& Hartmann, L. 1995, ApJS, 101, 117

Kenyon, S. J., Hartmann, L. W., Strom, K. M., \& Strom, S. E. 1990, AJ, 99, 869

Kirk, J. M., Ward-Thompson, D., Di Francesco, J., et al. 2009, ApJS, 185, 198

Knude, J., \& Hog, E. 1998, A\&A, 338, 897

Kryukova, E., Megeath, S. T., Gutermuth, R. A., et al. 2012, AJ, 144, 31

Lada, C. J., Lombardi, M., \& Alves, J. F. 2009, ApJ, 703, 52

Larson, R. B. 1969, MNRAS, 145, 271

Lee, C. W., Bourke, T. L., Myers, P. C., et al. 2009, ApJ, 693, 1290

Li, Z.-Y., \& Shu, F. H. 1997, ApJ, 475, 237

Lodato, G., \& Clarke, C. J. 2004, MNRAS, 353, 841

Lonsdale, C. J., Smith, H. E., Rowan-Robinson, M., et al. 2003, PASP, 115, 897

Machida, M. N., Inutsuka, S.-i., \& Matsumoto, T. 2008, ApJ, 676, 1088

Machida, M. N., Inutsuka, S.-i., \& Matsumoto, T. 2011, ApJ, 729, 42

Maury, A. J., André, P., Men'shchikov, A., Könyves, V., \& Bontemps, S. 2011, A\&A, 535, A77

Merín, B., Jørgensen, J., Spezzi, L., et al. 2008, ApJS, 177, 551

Myers, P. C. 2010, ApJ, 714, 1280

Myers, P. C. 2011, ApJ, 743, 98

Myers, P. C. 2012, ApJ, 752, 9

Myers, P. C., \& Ladd, E. F. 1993, ApJL, 413, L47

Nayakshin, S., \& Lodato, G. 2012, MNRAS, 426, 70

Neuhäuser, R., \& Forbrich, J. 2008, in Handbook of Star Forming Regions, Volume II: The Southern Sky, ed. B. Reipurth (ASP Monographs; San Francisco, CA: ASP), 735

Offner, S. S. R., \& McKee, C. F. 2011, ApJ, 736, 53

Oliveira, I., Merín, B., Pontoppidan, K. M., et al. 2009, ApJ, 691, 672

Oliveira, I., Pontoppidan, K. M., Merín, B., et al. 2010, ApJ, 714, 778

Padgett, D. L., Rebull, L. M., Stapelfeldt, K. R., et al. 2008, ApJ, 672, 1013 
Penston, M. V. 1969, MNRAS, 145, 457

Peterson, D. E., Caratti o Garatti, A., Bourke, T. L., et al. 2011, ApJS, 194, 43

Pezzuto, S., Elia, D., Schisano, E., et al. 2012, A\&A, 547, A54

Pfalzner, S., Tackenberg, J., \& Steinhausen, M. 2008, A\&A, 487, L45

Pineda, J. E., Arce, H. G., Schnee, S., et al. 2011, ApJ, 743, 201

Porras, A., Jørgensen, J. K., Allen, L. E., et al. 2007, ApJ, 656, 493

Price, D. J., Tricco, T. S., \& Bate, M. R. 2012, MNRAS, 423, L45

Rebull, L. M., Padgett, D. L., McCabe, C.-E., et al. 2010, ApJS, 186, 259

Rebull, L. M., Stapelfeldt, K. R., Evans, N. J., II, et al. 2007, ApJS, 171, 447

Rieke, G. H., Young, E. T., Engelbracht, C. W., et al. 2004, ApJS, 154, 25

Robitaille, T. P., Whitney, B. A., Indebetouw, R., Wood, K., \& Denzmore, P. 2006, ApJS, 167, 256

Romero, G. A., Schreiber, M. R., Cieza, L. A., et al. 2012, ApJ, 749, 79

Schnee, S., Di Francesco, J., Enoch, M., et al. 2012, ApJ, 745, 18

Shu, F. H. 1977, ApJ, 214, 488

Simon, J. B., Hawley, J. F., \& Beckwith, K. 2011, ApJ, 730, 94

Siringo, G., Kreysa, E., Kovács, A., et al. 2009, A\&A, 497, 945

Spezzi, L., Vernazza, P., Merín, B., et al. 2011, ApJ, 730, 65

Straižys, V., Černis, K., \& Bartašiute, S. 1996, BaltA, 5, 125

Tassis, K., \& Mouschovias, T. C. 2005, ApJ, 618, 783

Terebey, S., Shu, F. H., \& Cassen, P. 1984, ApJ, 286, 529
Vorobyov, E. I., \& Basu, S. 2005, ApJL, 633, L137

Vorobyov, E. I., \& Basu, S. 2006, ApJ, 650, 956

Vorobyov, E. I., \& Basu, S. 2010, ApJ, 719, 1896

Ward-Thompson, D., Di Francesco, J., Hatchell, J., et al. 2007, PASP, 119, 855

Weingartner, J. C., \& Draine, B. T. 2001, ApJ, 548, 296

Werner, M. W., Roellig, T. L., Low, F. J., et al. 2004, ApJS, 154, 1

Whitney, B. A., Wood, K., Bjorkman, J. E., \& Wolff, M. J. 2003, ApJ, 591, 1049

Whittet, D. C. B., Prusti, T., Franco, G. A. P., et al. 1997, A\&A, 327, 1194

Wilking, B. A., Gagné, M., \& Allen, L. E. 2008, in Handbook of Star Forming Regions, Volume II: The Southern Sky, ed. B. Reipurth (ASP Monographs; San Francisco, CA: ASP), 351

Wu, J., Dunham, M. M., Evans, N. J., II, Bourke, T. L., \& Young, C. H. 2007, AJ, 133,1560

Young, C. H., Jørgensen, J. K., Shirley, Y. L., et al. 2004, ApJS, 154, 396

Young, K. E., Enoch, M. L., Evans, N. J., II, et al. 2006, ApJ, 644, 326

Young, K. E., Harvey, P. M., Brooke, T. Y., et al. 2005, ApJ, 628, 283

Zhu, Z., Hartmann, L., \& Gammie, C. 2009a, ApJ, 694, 1045

Zhu, Z., Hartmann, L., Gammie, C., \& McKinney, J. C. 2009b, ApJ, 701, 620

Zhu, Z., Hartmann, L., Gammie, C. F., et al. 2010, ApJ, 713, 1143 